\title{
INTEGRABILITY OF INFINITE SUMS OF INDEPENDENT VECTOR-VALUED RANDOM VARIABLES $\left({ }^{1}\right)$
}

\author{
BY
}

\author{
NARESH C. JAIN AND MICHAEL B. MARCUS $\left(^{2}\right)$
}

\begin{abstract}
Let $B$ be a normed vector space (possibly a Banach space, but it could be more general) and $\left\{X_{n}\right\}$ a sequence of $B$-valued independent random variables on some probability space. Let $S_{n}=\sum_{j=1}^{n} X_{j}, M=\sup _{n}\left|S_{n}\right|$ and $S=\lim _{n} S_{n}$ is norm, whenever it exists. Assuming that $S$ exists or $M<\infty$ a.s. and given certain nondecreasing functions $\varphi$, we find conditions in terms of the distributions of $\left\|X_{n}\right\|$ such that $E(\varphi(M))$ or $E(\varphi(\|S\|))$ is finite.

Let $\left\{u_{n}\right\}$ be a sequence of elements in $B$ and $\left\{\epsilon_{n}\right\}$ a sequence of independent, identically distributed random variables such that $P\left\{\epsilon_{1}=1\right\}=$ $P\left\{\epsilon_{1}=-1\right\}=1 / 2$. We prove some comparison theorems which generalize the following contraction principle of Kahane: If $\left\{\lambda_{n}\right\}$ is a bounded sequence of scalars, then $\Sigma \epsilon_{n} u_{n}$ converges in norm a.s. (or is bounded a.s.) implies the corresponding conclusion for the series $\Sigma \lambda_{n} \epsilon_{n} u_{n}$. Some generalizations of this contraction principle have already been carried out by Hoffmann-Jфrgensen. All these earlier results are subsumed by ours.

Applications of our results are made to Gaussian processes, random Fourier series and other random series of functions.
\end{abstract}

1. Introduction. Let $(\Omega, F, P)$ be a probability space and $(B, B)$ a measurable space where $B$ is a linear space over the real field $R^{1}$ and $B$ a $\sigma$-algebra of subsets of $B$ which is compatible with the linear structure of $B$, i.e.

(1.1) $(x, y) \rightarrow x+y$ is a $B \mid B \times B$ measurable map from $B \times B$ into $B$, and

(1.2) for $\lambda \in R^{1}, x \in B$, the map $(\lambda, x) \rightarrow \lambda x$ from $R^{1} \times B$ into $B$ is a $B \mid B_{1} \times B$ measurable mapping, where $B_{1}$ denotes the Borel sets of $R^{1}$.

Definition 1.1. A function $X$ from $\Omega$ into $B$, which is $B \mid F$ measurable, will be called a $B$-valued random variable. If $X_{1}, \ldots, X_{n}$ are $B$-valued random variables, then we call them independent if, given $A_{1}, \ldots, A_{n} \in B$,

$$
P\left(X_{1} \in A_{1}, \ldots, X_{n} \in A_{n}\right)=\prod_{i=1}^{n} P\left(X_{i} \in A_{i}\right) \text {. }
$$

Received by the editors September 5, 1974.

AMS (MOS) subject classifications (1970). Primary 60G15, 60G50, 60B05.

Key words and phrases. Integrability, infinite series, Banach space valued random variables, convergence in norm, contraction principle, uniformly nondegenerate, Rademacher sequence.

(1) This research was supported in part by National Science Foundation grants.

$\left.{ }^{2}\right)$ On sabbatical leave at the Courant Institute of Mathematical Sciences, New York University, New York, when this research was carried out.

Copyright $\odot$ 1975, American Mathematical Society 
An infinite sequence of $B$-valued random variables on $(\Omega, F, P)$ is said to be a sequence of independent $B$-valued random variables if any finite subset of the sequence is independent.

DEFinition 1.2. A measurable norm on $(B, B)$ is a mapping $x \rightarrow\|x\|$ from $B$ to $[0, \infty)$ such that

(1.3) $\|x\|=0$ if and only if $x=0$,

(1.4) $\|x+y\| \leqslant\|x\|+\|y\|$,

(1.5) $\|\lambda x\|=|\lambda|\|x\|, \lambda$ real, and

(1.6) $\{x \in B:\|x\| \leqslant a\} \in B$ for all $a \in R^{1}$.

If $B$ is a Banach space, then its norm is a measurable norm in the sense of Definition $1.2, B$ is taken to consist of the Borel sets.

If $X$ is a $B$-valued random variable on $(\Omega, F, P)$ and $\|\cdot\|$ is a measurable norm on $B$, then $\|X\|$ is a real-valued random variable on $(\Omega, F, P)$ in the usual sense. From now on we will assume that $B$ has a measurable norm $\|\cdot\|$.

Let $\left\{X_{n}, n \geqslant 1\right\}$ be a sequence of $B$-valued independent random variables on $(\Omega, F, P)$. We will always write

(1.7) $S_{n}=\sum_{j=1}^{n} X_{j}$,

(1.8) $M_{n}=\sup _{1 \leqslant j \leqslant n}\left\|S_{j}\right\|$,

(1.9) $N_{n}=\sup _{1 \leqslant j \leqslant n}\left\|X_{j}\right\|$,

(1.10) $M=\sup _{j \geqslant 1}\left\|S_{j}\right\|$,

(1.11) $N=\sup _{j \geqslant 1}\left\|X_{j}\right\|$,

and if $S_{n}$ converges in norm, then

(1.12) $S=\sum_{j=1}^{\infty} X_{j}$.

When $B=R^{1}$ the question of convergence of $S_{n}$ has been very satisfactorily answered by Kolmogorov's three-series theorem. However, there are no comprehensive results when, e.g., $X_{n}$ are independent Banach space-valued random variables. Extensive results exist in special cases, like the study of random trigonometric series or Gaussian processes (for references see [4], [5]), but even in these cases results are not complete.

In this article we will assume that $S$ exists, or that $M<\infty$ a.s. Then given certain nondecreasing functions $\varphi$ we find conditions in terms of $\varphi$ and the distributions of $\left\|X_{n}\right\|$ such that $E(\varphi(M))$ or $E(\varphi(\|S\|))$ is finite, where $E$ denotes expectation. Some of our results appear to be new even when $B=R^{1}$ (see, for example, Theorem 3.6).

For earlier work in this direction, we mention the result of Landau and Shepp [6], who showed that if $\left\{X_{n}\right\}$ is a sequence of real-valued Gaussian random variables such that $Z=\sup _{n}\left|X_{n}\right|<\infty$ a.s., then $E\left(\exp \left(\epsilon Z^{2}\right)\right)<\infty$ for some $\epsilon>0$. Fernique [2] obtained a similar result. Kahane [5] proves results of this nature for random trigonometric series involving Rademacher sequences, and Hoffmann-J $\phi$ rgensen [3], whose paper stimulated our present research, considers the 
same question we do when $\varphi$ is a power. Most of these results, often in stronger form, will be rederived. These references will be discussed in greater detail in appropriate context later on.

In $\S 2$ we give some more notation and preliminary lemmas. $\S 3$ contains the main results. An important special case is discussed in $\S 4$, where $X_{n}$ is of the form $v_{n} Y_{n}, v_{n}$ a nonrandom element of $B$ and $Y_{n}$ a real-valued random variable. A counterexample given in this section shows how drastically the situation differs from the usual real-valued case.

In $\S 5$ we also consider the case where $X_{n}$ is of the form $v_{n} Y_{n}$. What we show is essentially this. If $\sup _{n}\left\|\Sigma_{k=1}^{n} v_{k} Y_{k}\right\|<\infty$ a.s. and if $\left\{\xi_{n}\right\}$ is a sequence of independent symmetric random variables that are smaller than the $\left\{Y_{n}\right\}$ in some appropriate sense then $\sup _{n}\left\|\Sigma_{k=1}^{n} v_{k} \xi_{k}\right\|<\infty$ a.s. also. A similar result holds if the first series converges. Furthermore, results of the type

$$
E\left[\varphi\left(\sup _{n}\left\|\sum_{k=1}^{n} v_{k} \xi_{k}\right\|\right)\right] \leqslant E\left[\varphi\left(\sup _{n}\left\|\sum_{k=1}^{n} v_{k} Y_{k}\right\|\right)\right],
$$

for certain convex functions $\varphi$, also hold. These results are actually extensions of the contraction principle of Kahane [5].

Applications of the results of $\S \S 3,4$ and 5 to Gaussian processes, random Fourier series and other random series of functions are given in $\S 6$.

Note. After these results were submitted for publication, [3] appeared in the form of [3a] with certain results in improved form over those of [3]. Consequently some special cases of our results are now equivalent to the results in [3a].

2. Preliminaries and notation. We will add to the definitions and notation given in the previous section. Some well-known results, that will be needed later, will also be given here.

Definition 2.0. A $B$-valued random variable $X$ on $(\Omega, F, P)$ is said to be symmetric if

$$
P(X \in A)=P(-X \in A), \quad A \in B .
$$

For symmetric random variables we have the following inequality of $P$. Lévy (see $[5$, p. 12] for a proof).

LEMMA 2.1. Let $\left\{X_{n}, n \geqslant 1\right\}$ be a sequence of symmetric, $B$-valued, independent random variables such that $S=\Sigma_{j=1}^{\infty} X_{j}$ exists, then, for all $\lambda>0$,

$$
P(M>\lambda) \leqslant 2 P(\|S\|>\lambda) \text {. }
$$

Note that one can take $X_{j}=0$ for $j>n$, then (2.2) applies to $M_{n}$ and $S_{n}$ in place of $M$ and $S$, respectively. 
The distribution function $F$ of a real-valued random variable $X$ on $(\Omega, F, P)$ is defined by

$$
F(x)=P(X \leqslant x) .
$$

For a real-valued measurable function $g$ on $R^{1}, G$ a right-continuous function with bounded variation on compact sets, $\int g d G$ will always denote the integral of $g$ with respect to the measure $\mu$ determined by $G$ on $R^{1}$ by setting $\mu(a, b]=$ $G(b)-G(a)$.

If $\varphi$ is a nondecreasing, continuous function on $R^{1}, F$ a distribution function (right-continuous, nonnegative, nondecreasing, $\lim _{x \rightarrow-\infty} F(x)=0, \lim _{x \rightarrow \infty} F(x)$ $=1$ ) and $Q=1-F$, then for $-\infty<a \leqslant b<\infty$ we have the well-known integration by parts formula

$$
\int \varphi I_{(a, b]} d F=\varphi(a) Q(a)-\varphi(b) Q(b)+\int_{a}^{b} Q d \varphi
$$

where $I_{(a, b]}(x)=1$ or 0 according as $x \in(a, b]$ or $x \notin(a, b]$. If the limit as $b \rightarrow \infty$ exists for the left side in (2.4), then $\varphi(b) Q(b) \rightarrow 0$, and consequently $\lim _{b \rightarrow \infty} \int_{a}^{b} Q d \varphi$ exists. In this case we also have

$$
\int_{(a, \infty)} \varphi d F=\varphi(a) Q(a)+\int_{a}^{\infty} Q d \varphi .
$$

If $\varphi \geqslant 0, \varphi(0)=0$, and $X$ is a nonnegative finite random variable with distribution function $F$, then

$$
E(\varphi(X))=\int_{(0, \infty)} \varphi d F=\int_{0}^{\infty} Q d \varphi
$$

by (2.5).

Notation. We will write $I[A]$ to mean the indicator of the set $A$. $\Phi$ will denote the class of all finite-valued nonnegative, nondecreasing, continuous, functions on $[0, \infty)$, which are not identically zero. Let $\Phi_{0}=\{\varphi \in \Phi: \varphi(0)=0\}$.

For $\varphi \in \Phi$, we shall write

$$
\varphi_{a}(x)=\varphi(x) I[x>a] .
$$

The abbreviation "a.s." stands for "almost surely". $(\Omega, F, P)$ will be used generically for a probability space. $E$ will denote the integration operator on $L^{1}(\Omega, F, P)$.

3. Integrability of $\varphi(M)$ and $\varphi(\|S\|)$. Let $\left\{X_{n}, n \geqslant 1\right\}$ be a sequence of independent $B$-valued random variables on $(\Omega, F, P)$. Let $\varphi \in \Phi$. The main results of this section, Theorems 3.3, 3.6, 3.8 and 3.11, give conditions in terms of $\varphi$ and the distributions of $\left\|X_{n}\right\|$ under which $\varphi(M)$ and $\varphi(\|S\|)$ are integrable.

The following lemma is part of Theorem 3.3, except that more restrictive conditions on $\varphi$ are imposed there. 
LEMMA 3.1. Let $\left\{Y_{n}, n \geqslant 1\right\}$ be a sequence of independent nonnegative random variables on $(\Omega, F, P)$. Let $\varphi \in \Phi$. If $N=\sup _{n} Y_{n}$, then

(3.1) $N<\infty$ a.s. and $E(\varphi(N))<\infty \Leftrightarrow a>0$ such that $\sum_{n=1}^{\infty} E\left(\varphi_{a}\left(Y_{n}\right)\right)<\infty$.

REMARK 3.2. If $\varphi \equiv 1$, then (3.1) reads as

$$
N<\infty \text { a.s. } \Leftrightarrow a>0 \text {, such that } \sum_{n=1}^{\infty} P\left(Y_{n}>a\right)<\infty \text {. }
$$

Proof of Lemma 3.1. If $\varphi$ is bounded, then, using the facts that $\varphi$ is nondecreasing and not identically zero, it is easily seen that (3.1) reduces to (3.2). We prove (3.2) first. Using independence,

$$
\begin{aligned}
P(N>u) & =\sum_{j=1}^{\infty} P\left(Y_{1} \leqslant u, \ldots, Y_{j-1} \leqslant u, Y_{j}>u\right) \\
& =\sum_{j=1}^{\infty}\left(1-\alpha_{j}(u)\right) P\left(Y_{j}>u\right),
\end{aligned}
$$

where $\alpha_{j}(u)=P\left(\bigcup_{k=1}^{j-1}\left[Y_{k}>u\right]\right) / P(N>u)$. If $N<\infty$ a.s., then $\exists u_{0}$ such that $P\left(N>u_{0}\right) \leqslant 1 / 2$. Therefore from (3.3) we have $\Sigma P\left(Y_{j}>u_{0}\right)<\infty$. Conversely if the right side in (3.2) holds, then $\Sigma_{j} P\left(Y_{j}>u\right) \rightarrow 0$ as $u \rightarrow \infty$, hence from (3.3) we then have $\lim _{u \rightarrow \infty} P(N>u)=0$. Hence $N<\infty$ a.s.

For the general case, let $N_{k}=\max _{1<j<k} Y_{j}$. Then using independence, we have

$$
P\left(N_{k}>u\right)=1-\prod_{n=1}^{k}\left(1-P\left(Y_{n}>u\right)\right) \geqslant 1-\exp \left(-\sum_{n=1}^{k} P\left(Y_{n}>u\right)\right),
$$

hence letting $k / \infty$, we get

$$
P(N>u) \geqslant 1-\exp \left(-\sum_{n=1}^{\infty} P\left(Y_{n}>u\right)\right) \text {. }
$$

Assuming the left side in (3.1), we have $\sum_{n=1}^{\infty} P\left(Y_{n}>a\right)<\infty$ for some $a>0$ by (3.2). Hence

$$
\lim _{u \rightarrow \infty} \sum_{n=1}^{\infty} P\left(Y_{n}>u\right)=0
$$

and there exists $a>0$ such that $\Sigma_{n=1}^{\infty} P\left(Y_{n}>a\right) \leqslant 1 / 2$, we have

$$
\begin{aligned}
\frac{1}{2} \int_{a}^{\infty} \sum_{n=1}^{\infty} P\left(Y_{n}>u\right) d \varphi(u) \\
\leqslant \int_{a}^{\infty}\left[1-\exp \left(-\sum_{n=1}^{\infty} P\left(Y_{n}>u\right)\right)\right] d \varphi(u) \leqslant E(\varphi(N))
\end{aligned}
$$


by (3.4) and (2.5). Also by (2.5) we get

$$
\sum_{n=1}^{\infty} \int_{a}^{\infty} P\left(Y_{n}>u\right) d \varphi(u)=\sum_{n=1}^{\infty} E\left(\varphi_{a}\left(Y_{n}\right)\right)-\varphi(a) \sum_{n=1}^{\infty} P\left(Y_{n}>a\right) .
$$

Combining (3.6) and (3.7) gives the right side in (3.1).

For the converse, observe that since $\varphi$ is continuous we have

$$
\varphi_{a}(N) \leqslant \sum_{n=1}^{\infty} \varphi_{a}\left(Y_{n}\right)
$$

Since $\varphi$ is nondecreasing and not identically zero, $\Sigma_{n=1}^{\infty} E\left(\varphi_{a}\left(Y_{n}\right)\right)<\infty$ implies the right side in (3.2), hence

$$
\sum_{n=1}^{\infty} E\left(\varphi_{a}\left(Y_{n}\right)\right)<\infty \Rightarrow N<\infty \text { a.s. and } E\left(\varphi_{a}(N)\right)<\infty .
$$

The result now follows by observing that $E(\varphi(N)) \leqslant \varphi(a)+E\left(\varphi_{a}(N)\right)$.

The assumptions on $\varphi$ in the following theorem are satisfied by all continuous regularly varying functions, i.e. functions of the form $\varphi(u)=u^{p} \psi(u), p \geqslant 0$, $\psi$ a continuous slowly varying function near $\infty$. The exponential case is given in Theorem 3.8.

THEOREM 3.3. Suppose $M=\sup _{n}\left\|S_{n}\right\|<\infty$ a.s., and let $N=\sup _{n}\left\|X_{n}\right\|$. If $\varphi \in \Phi$ and satisfies $\varphi(4 u) \leqslant c \varphi(u)$ for some $c>0$ then the following statements are equivalent:

(a) $E(\varphi(N))<\infty$;

(b) $E(\varphi(M))<\infty$;

(c) $\exists a>0$ such that $\Sigma_{n=1}^{\infty} E\left(\varphi_{a}\left(\left\|X_{n}\right\|\right)\right)<\infty$, where $\varphi_{a}(x)=\varphi(x) I[x>a]$.

Furthermore, if $S=\Sigma_{n=1}^{\infty} X_{n}$ converges a.s., then each of the above statements is equivalent to

(d) $E(\varphi(\|S\|))<\infty$ and, for $\varphi \in \Phi_{0}, \lim _{n \rightarrow \infty} E\left(\varphi\left(\left\|S-S_{n}\right\|\right)\right)=0$.

We shall need the following lemma from Hoffmann-J $\phi$ rgensen ([3, Theorem 3.1], [3a]). The proof is a modification of the argument in Kahane [5, p. 16]. We reproduce the proof for completeness.

LEMMA 3.4. Let $X_{n}$ be symmetric, independent, $B$-valued random variables on $(\Omega, F, P)$. Let $F_{k}(u)=P\left(\left\|S_{k}\right\|>u\right), G(u)=P(N>u)$. Then for $t>0, s>0$

$$
F_{k}(2 t+s) \leqslant G(s)+4 F_{k}^{2}(t) .
$$

Proof. Let $T=\inf \left\{n \geqslant 1:\left\|S_{n}\right\|>t\right\}$. Since $\left\|S_{k}\right\|>2 t+s$ implies $T \leqslant k$, we have 


$$
F_{k}(2 t+s)=\sum_{j=1}^{k} P\left(T=j,\left\|S_{k}\right\|>2 t+s\right) .
$$

If $T=j$ and $\left\|S_{k}\right\|>2 t+s$, then $\left\|S_{j-1}\right\|<t$, and $\left\|S_{k}-S_{j}\right\|=\left\|S_{k}-S_{j-1}-X_{j}\right\|$ $\geqslant\left\|S_{k}\right\|-\left\|S_{j-1}\right\|-\left\|X_{j}\right\| \geqslant t+s-N$. Hence

$$
\begin{gathered}
P\left(T=j,\left\|S_{k}\right\|>2 t+s\right) \leqslant P\left(T=j,\left\|S_{k}-S_{j}\right\| \geqslant t+s-N\right) \\
\leqslant P\left(T=j,\left\|S_{k}-S_{j}\right\| \geqslant t\right)+P(T=j, N>s) \\
=P(T=j) P\left(\left\|S_{k}-S_{j}\right\|>t\right)+P(T=j, N>s)
\end{gathered}
$$

using independence at the last step. Summing on $j$ and using (3.7') we get

$$
F_{k}(2 t+s) \leqslant P(N>s)+\sum_{j=1}^{k} P(T=j) P\left(\left\|S_{k}-S_{j}\right\|>t\right) .
$$

By Lemma 2.1 we have

$$
P\left(\left\|S_{k}-S_{j}\right\|>t\right) \leqslant 2 P\left(\left\|S_{k}\right\|>t\right)
$$

and

$$
\sum_{j=1}^{k} P(T=j)=P\left(\max _{1 \leqslant j \leqslant k}\left\|S_{j}\right\|>t\right) \leqslant 2 P\left(\left\|S_{k}\right\|>t\right) .
$$

Combining (3.9), (3.10) and (3.11) we get (3.6').

Proof OF Theorem 3.3. We have already shown $(\mathrm{a}) \Leftrightarrow$ (c) under less restrictive conditions on $\varphi$. To see that (b) $\Rightarrow\left(\right.$ a), note that $\left\|X_{n}\right\| \leqslant\left\|S_{n}\right\|+$ $\left\|S_{n-1}\right\|$. Hence taking the sup over $n \geqslant 1$, we have $N \leqslant 2 M$, and so $\varphi(N) \leqslant$ $\varphi(2 M) \leqslant \varphi(4 M) \leqslant c \varphi(M)$. We now show that (a) $\Rightarrow$ (b). Note that we may assume $\varphi(0)=0$ without any loss of generality. We first assume that the $X_{j}$ are symmetric random variables. Then by Lemma 3.4 we have

$$
\int_{0}^{\infty} F_{k}(4 t) d \varphi(t) \leqslant 4 \int_{0}^{\infty} F_{k}^{2}(t) d \varphi(t)+\int_{0}^{\infty} G(t) d \varphi(t),
$$

where $F_{k}(u)=P\left(\left\|S_{k}\right\|>u\right), G(u)=P(N>u)$. Since $\varphi(0)=0$, by integration by parts the left side in (3.12) equals $E\left(\varphi\left(\left\|S_{k}\right\| / 4\right)\right)$, which dominates $c^{-1} E\left(\varphi\left(\left\|S_{k}\right\|\right)\right)$ since $\varphi(4 t) \leqslant c \varphi(t)$. Hence (3.12) gives

$$
\int_{0}^{\infty}\left(c^{-1} F_{k}(t)-4 F_{k}^{2}(t)\right) d \varphi(t) \leqslant E(\varphi(N)) .
$$

Since $M<\infty$ a.s. by assumption, there exists a $t_{0}$ such that for $t \geqslant t_{0}, P(M>t)$ $\leqslant(8 c)^{-1}$. Hence for $t \geqslant t_{0}, F_{k}(t) \leqslant(8 c)^{-1}$ for all $k \geqslant 1$. Hence

$$
\int_{t_{0}}^{\infty}\left(c^{-1} F_{k}(t)-4 F_{k}^{2}(t)\right) d \varphi(t) \geqslant \frac{1}{2 c} \int_{t_{0}}^{\infty} F_{k}(t) d \varphi(t) .
$$


Combining this with (3.13), we get

$$
E\left(\varphi\left(\left\|S_{k}\right\|\right)\right) \leqslant \varphi\left(t_{0}\right)+2 c E(\varphi(N)) .
$$

By Lemma 2.1

$$
P\left(\max _{1 \leqslant n<k}\left\|S_{n}\right\|>t\right) \leqslant 2 P\left(\left\|S_{k}\right\|>t\right) .
$$

This together with (3.15) gives

$$
E\left(\varphi\left(\max _{1 \leqslant n<k}\left\|S_{n}\right\|\right)\right) \leqslant 2 \varphi\left(t_{0}\right)+4 c E(\varphi(N)) .
$$

This shows $(a) \Rightarrow(b)$ in the symmetric case. In the general case, we use symmetrization. Let $\left\{X_{n}, n \geqslant 1\right\}$ be the given sequence and $\left\{X_{n}^{\prime}, n \geqslant 1\right\}$ an independent copy. Let $N^{\prime}=\sup _{n}\left\|X_{n}^{\prime}\right\|$ and $M^{\prime}=\sup _{n}\left\|S_{n}^{\prime}\right\|, S_{n}^{\prime}=X_{1}^{\prime}+\cdots+X_{n}^{\prime}$. Note that

$$
\begin{aligned}
\varphi\left(\operatorname{Sup}_{n}\left\|X_{n}-X_{n}^{\prime}\right\|\right) & \leqslant \varphi\left(N+N^{\prime}\right) \leqslant \varphi\left(2 \max \left(N, N^{\prime}\right)\right) \\
& \leqslant c \varphi\left(\max \left(N, N^{\prime}\right)\right) \leqslant c\left[\varphi(N)+\varphi\left(N^{\prime}\right)\right] .
\end{aligned}
$$

Hence

$$
E(\varphi(N))<\infty \Rightarrow E\left(\varphi\left(\operatorname{Sup}_{n}\left\|X_{n}-X_{n}^{\prime}\right\|\right)\right)<\infty .
$$

Applying our result for the symmetric case, we have

$$
E\left(\varphi\left(\sup _{n}\left\|X_{n}-X_{n}^{\prime}\right\|\right)\right)<\infty \Rightarrow E\left(\varphi\left(\sup _{n}\left\|S_{n}-S_{n}^{\prime}\right\|\right)\right)<\infty .
$$

It remains to check that

$$
E\left(\varphi\left(\operatorname{Sup}_{n}\left\|S_{n}-S_{n}^{\prime}\right\|\right)\right)<\infty \Rightarrow E(\varphi(M))<\infty
$$

To see this, let $\varphi=0$ on $(-\infty, 0)$, then

$$
E\left(\varphi\left(\operatorname{Sup}_{n}\left\|S_{n}-S_{n}^{\prime}\right\|\right)\right) \geqslant E\left(\varphi\left(M-M^{\prime}\right)\right)
$$

and by the independence of $M, M^{\prime}$ and Fubini's theorem, we have

$$
E\left(\varphi\left(M-M^{\prime}\right)\right)<\infty \Rightarrow \exists y \geqslant 0 \text { such that } E(\varphi(M-y))<\infty .
$$

Since $M-y \geqslant M / 2$ on the set $\{M>2 y\}$, we conclude that

$$
E\left(\varphi\left(\operatorname{Sup}_{n}\left\|S_{n}-S_{n}^{\prime}\right\|\right)\right)<\infty \Rightarrow \int_{\{M>2 y\}} \varphi(M / 2) d P<\infty .
$$


Since $E(\varphi(M / 2)) \leqslant \varphi(y)+\int_{\{M>2 y\}} \varphi(M / 2) d P$, and by assumption $\varphi(4 u) \leqslant$ $c \varphi(u)$, it follows that

$$
E\left(\varphi\left(\operatorname{Sup}_{n}\left\|S_{n}-S_{n}^{\prime}\right\|\right)\right)<\infty \Rightarrow E(\varphi(M))<\infty .
$$

Hence (3.18), (3.19) and (3.24) prove (a) $\Rightarrow(b)$ without the assumption of symmetry.

It remains to prove the last part of the theorem. Since $\|S\| \leqslant M$, we have that (b) $\Rightarrow E(\varphi(\|S\|))<\infty$. In the symmetric case Lemma 2.1 gives $P(M>u) \leqslant$ $2 P(\|S\|>u)$, and so $E(\varphi(\|S\|))<\infty \Rightarrow(b)$. In the general case the validity of this conclusion follows via symmetrization. Hence (b) $\Leftrightarrow E(\varphi(\|S\|))<\infty$. Since $2 M \geqslant\left\|S_{n}-S\right\| \rightarrow 0$ a.s., (b) implies (by the dominated convergence theorem) that when $\varphi \in \Phi_{0}, E\left(\varphi\left(\left\|S_{n}-S\right\|\right)\right) \rightarrow 0$ as $n \rightarrow \infty$. This completes the proof of the theorem.

If $\varphi(u)=u^{p}$ for some $p \geqslant 0$, then, as we already observed, $\varphi$ satisfies the conditions of Theorem 3.3, and we get the following important corollary. This should be compared with Hoffmann-J $\phi$ rgensen's Theorem 3.1 [3], where he shows that $E\left(N^{p}\right)<\infty, p>0 \Rightarrow E\left(M^{q}\right)<\infty$ for $0 \leqslant q<p$. (The improved version of Theorem 3.1 in [3a] also gives parts (a) and (b) of this corollary.)

Corollary 3.5. Suppose $M<\infty$ a.s. If $p>0$, then the following are equivalent:

(a) $E\left(N^{p}\right)<\infty$;

(b) $E\left(M^{p}\right)<\infty$;

(c) $\sum_{n=1}^{\infty} E\left(\left\|X_{n}\right\|^{p} I\left[\left\|X_{n}\right\|>a\right]\right)<\infty$, for some $a>0$.

Furthermore, if $\Sigma X_{n}$ converges a.s. and $S$ represents this sum, then each of the above statements is equivalent to

(d) $E\left(\|S\|^{p}\right)<\infty$ and $E\left(\left\|S_{n}-S\right\|^{p}\right) \rightarrow 0$ as $n \rightarrow \infty$.

If the $X_{j}$ 's are independent, real-valued, random variables, then combining Theorem 3.3 with Kolmogorov's three-series theorem, we get

THEOREM 3.6. Let $\left\{X_{j}, j \geqslant 1\right\}$ be a sequence of real-valued independent random variables on some probability space $(\Omega, F, P)$. If $\varphi \in \Phi$ and satisfies $\varphi(4 u) \leqslant c \varphi(u)$ for some $c>0$ and all $u \geqslant 0$, then

$\Sigma X_{j}$ converges a.s.,

$E\left[\varphi\left(\left|\Sigma X_{j}\right|\right)\right]<\infty$,

and if $\varphi \in \Phi_{0}$

$\lim _{n \rightarrow \infty} E\left[\varphi\left(\left|\Sigma_{j=n}^{\infty} X_{j}\right|\right)\right]=0$

if and only if the following three series converge for some $c>0$ :

(a) $\Sigma_{j} E\left(X_{j} I\left[\left|X_{j}\right| \leqslant c\right]\right)$, 
(b) $\Sigma_{j}\left\{E\left(X_{j}^{2} I\left[\left|X_{j}\right| \leqslant c\right]\right)-E^{2}\left(X_{j} I\left[\left|X_{j}\right| \leqslant c\right]\right)\right\}$,

(c) $\Sigma_{j} E\left(\varphi\left(\left|X_{j}\right|\right) I\left[\left|X_{j}\right|>c\right]\right)$.

REMARK 3.7. When $\varphi=1$, this theorem reduces to the three-series theorem.

Proof. First assume (a), (b), (c). Since $\varphi$ is not identically zero, the other conditions on $\varphi$ imply that $\varphi(u)>0$ for $u>0$. Since

$$
\sum_{j} E\left(\varphi\left(\left|X_{j}\right|\right) I\left[\left|X_{j}\right|>c\right]\right) \geqslant \varphi(c) \sum_{j} P\left(\left|X_{j}\right|>c\right),
$$

(c) implies that $\Sigma_{j} P\left(\left|X_{j}\right|>c\right)<\infty$. This together with (a) and (b) imply that $\Sigma X_{j}$ converges by the three-series theorem. By Theorem 3.1 the condition (c) above then implies that $E\left(\varphi\left(\left|\Sigma X_{j}\right|\right)\right)<\infty$, and for $\varphi \in \Phi_{0}, E\left(\varphi\left(\left|\sum_{j=n}^{\infty} X_{j}\right|\right)\right) \rightarrow 0$ as $n \rightarrow \infty$. Conversely, if $\Sigma X_{j}$ converges, then (a) and (b) follow from the threeseries theorem and $E\left(\varphi\left(\left|\Sigma X_{j}\right|\right)\right)<\infty$ implies (c) by Theorem 3.3.

We next consider the case when $\varphi(x)=\exp (x)$. In this case it turns out that we do not quite get the expected analogue of Theorem 3.3 but Theorem 3.8 is satisfactory for a number of interesting cases.

THEOREM 3.8. Let $\left\{X_{n}, n \geqslant 1\right\}$ be independent $B$-valued random variables on $(\Omega, F, P)$. Suppose $M=\sup _{n}\left\|S_{n}\right\|<\infty$ a.s., and for some $a>0, \alpha>0$ and $\delta>0$

$$
\sum_{n=1}^{\infty} E\left\{\exp \left(\alpha\left\|X_{n}\right\| \log ^{1+\delta}\left\|X_{n}\right\|\right) I\left[\left\|X_{n}\right\|>a\right]\right\}<\infty .
$$

Then there exists an $\epsilon>0$ such that $E[\exp (\epsilon M)]<\infty$.

For convenience in writing we will prove the theorem for $\delta=1$; it should be obvious how to modify the argument for any $\delta>0$. Actually, one can replace $\log ^{1+\delta}\left\|X_{n}\right\|$ by a more general nondecreasing function of $\left\|X_{n}\right\|$. We will not go into that.

We first prove the following lemma.

LEMMA 3.9. Let $\left\{X_{n}, n \geqslant 1\right\}$ be independent $B$-valued random variables on $(\Omega, F, P)$. Suppose $M=\sup _{n}\left\|S_{n}\right\|<\infty$ a.s. and that (3.25) holds. Let $F_{k}(u)=$ $P\left(\left\|S_{k}\right\|>u\right)$ and $\gamma(u)=E\left[\exp \left(\alpha N \log ^{2} N\right) I(N>u)\right]$. Then for all $\epsilon>0, t>0$ and $k \geqslant 1$ we have

$$
\begin{aligned}
E\left(\exp \left(\epsilon\left\|S_{k}\right\|\right)\right) \leqslant & \exp (4 \epsilon t)+\sum_{n=0}^{\infty} \exp \left(\epsilon 2^{n+3} t\right)\left(8 F_{k}(t / 4)\right)^{2^{n+1}} \\
& +\sum_{n=0}^{\infty} \exp \left(\epsilon t 2^{n+3}\right) \sum_{s=1}^{n+1}\left(8^{2} \gamma\left(t s^{-2} 2^{s-2}\right)\right)^{2^{n-s+1}} \\
& \cdot \exp \left(-\alpha t s^{-2} 2^{n-1} \log ^{2}\left(t s^{-2} 2^{s-2}\right)\right)
\end{aligned}
$$


Proof. By Lemma 3.1 the assumption (3.25) is equivalent to

$$
E\left(\exp \left(\alpha N \log ^{2} N\right)\right)<\infty .
$$

From this we conclude that for $u>0$

$$
G(u)=P(N>u) \leqslant \gamma(u) \exp \left(-\alpha u \log ^{2} u\right) .
$$

Applying Lemma 3.4 with $t=2^{n} t_{1}$ and $s=(n+1)^{-2} 2^{n+1} t_{1}$ we get

$$
F_{k}\left[2^{n-1}\left(1+(n+1)^{-2}\right) t_{1}\right] \leqslant 4 F_{k}^{2}\left(2^{n} t_{1}\right)+G\left(2^{n+1}(n+1)^{-2} t_{1}\right) .
$$

Define $t_{2}$ by tile equation $2^{n} t_{1}=2^{n}\left(1+1 / n^{2}\right) t_{2}$, then

$$
F_{k}\left(2^{n} t_{1}\right) \leqslant 4 F_{k}^{2}\left(2^{n-1} t_{2}\right)+G\left(2^{n} n^{-2} t_{2}\right) \text {. }
$$

Substituting this in (3.29) and continuing recursively (making repeated use of the inequality $\left.(4 x+y)^{n} \leqslant(8 x)^{n}+(8 y)^{n}, x \geqslant 0, y \geqslant 0\right)$ we get

$$
\begin{aligned}
& F_{k}\left[2^{n+1}\left(1+(n+1)^{-2}\right) t_{1}\right] \\
& \leqslant\left(8 F_{k}\left(t_{n+1}\right)\right)^{2^{n+1}}+\sum_{j=0}^{n}\left[8^{2} G\left(2^{n+1-j} t_{j+1}(n+1-j)^{-2}\right)\right]^{2^{j}},
\end{aligned}
$$

where $t_{j}$ is given by

$$
\begin{aligned}
t_{j+1} & =\left(1-\frac{1}{1+(n+1-j)^{2}}\right) t_{j} \\
& =t_{1}\left(1-\frac{1}{1+n^{2}}\right)\left(1-\frac{1}{1+(n-1)^{2}}\right) \cdots\left(1-\frac{1}{1+(n+1-j)^{2}}\right) \geqslant \frac{t_{1}}{4} .
\end{aligned}
$$

Hence we get from (3.30) and (3.31)

$$
F_{k}\left(2^{n+2} t_{1}\right) \leqslant\left(8 F_{k}\left(t_{1} / 4\right)\right)^{2^{n+1}}+\sum_{s=1}^{n+1}\left[8 G\left(t_{1} 2^{s-2} s^{-2}\right)\right]^{2^{n-s+1}}
$$

In subsequent uses of (3.32) we will substitute $t$ for $t_{1}$. For $\epsilon>0$, we have

$$
\begin{aligned}
E\left(\exp \left(\epsilon\left\|S_{k}\right\|\right)\right) & \leqslant-\sum_{n=2}^{\infty} \int_{\left(2^{n} t, 2^{n+1} t\right]} \exp (\epsilon x) d F_{k}(x)+\exp (4 \epsilon t) \\
& \leqslant \exp (4 \epsilon t)+\sum_{n=0}^{\infty} \exp \left(\epsilon 2^{n+3} t\right) F_{k}\left(2^{n+2} t\right) .
\end{aligned}
$$

The estimate for $G(u)$ given in (3.28) is now used in (3.32), and the resulting estimate for $F_{k}\left(2^{n+2} t\right)$ is then used in (3.33) to get (3.26). This completes the proof of the lemma.

Proof of Theorem 3.8. We will establish this only in the symmetric 
case. The general case is then established by the familiar symmetrization procedure. The proof consists of showing that one can pick $t$ sufficiently large and $\epsilon$ sufficiently small, independent of $k$, such that the right side in (3.26) is finite. Note that $\gamma(u) \leqslant \gamma(0)$, where $\gamma(u)$ is defined in the statement of Lemma 3.9. Setting $\gamma_{1}=\max \left(8^{2} \gamma(0), 2\right)$, we can dominate the right side in (3.26) by

$$
\begin{aligned}
\exp (4 \epsilon t) & +\sum_{n=0}^{\infty} \exp \left(\epsilon t 2^{n+3}\right)\left(8 F_{k}(t / 4)\right)^{2^{n+1}} \\
& +\sum_{n=0}^{\infty} \exp \left(\epsilon t 2^{n+3}\right) \cdot \gamma_{1}^{2^{n+1}} \cdot \exp \left(-\alpha t 2^{n-2}\right)
\end{aligned}
$$

provided $t$ is chosen large enough so $s^{-2} \log ^{2}\left(t s^{-2} 2^{s-2}\right) \geqslant 2^{-1}$. Clearly such a choice of $t$ does not depend on $k$. Now pick $t$, larger if necessary, so that $\gamma_{1} \exp (-\alpha t / 8) \leqslant 1 / 2$ and $8 P(M>t / 4) \leqslant 1 / 2$. (Here we use $M<\infty$ a.s.) Such a choice of $t$ having been made, pick $\epsilon>0$ sufficiently small so that $\exp (4 \epsilon t)<$ 5/4. For such $\epsilon>0, t>0$, it is clear that the expression in (3.34) is finite. Hence there exists $0 \leqslant A<\infty$, and $\epsilon>0$, both independent of $k$, such that

$$
E\left(\exp \left(\epsilon\left\|S_{k}\right\|\right)\right) \leqslant A \text {. }
$$

By Lemma 2.1 and integration by parts this implies $E\left(\exp \left(\epsilon M_{k}\right)\right) \leqslant 2 A$, independently of $k$. Using the monotone convergence theorem the proof is completed.

REMARK 3.10. Even when (3.25) is not satisfied it is possible to obtain results of the kind $E[\varphi(M)]<\infty$ for $\varphi$ of exponential type. For instance suppose (3.25) is satisfied only when $\left\|X_{n}\right\|$ is replaced by $\left\|X_{n}\right\|^{\alpha}$ for some $0<\alpha<1$.

Under these conditions we could show $E\left(\exp \left(\epsilon M^{\alpha}\right)\right)<\infty$. The proof begins with Lemma 3.4 but the recursion is carried out differently. The choices of $t$ and $s$ in (3.26) determine the size of the terms involving $G$ which in turn determine the necessary conditions for the theorem.

The following result holds if one imposes even more restrictive conditions on the $X_{n}$. We are indebted to the referee for the proof of this theorem that is given here. It is considerably simpler than our original proof.

THEOREM 3.11. Let $\left\{X_{n}, n \geqslant 1\right\}$ be independent $B$-valued random variables on $(\Omega, F, P)$. If there exists a sequence $\left\{a_{n}\right\}$ of positive numbers such that

$$
\sup _{\omega}\left\|X_{n}(\omega)\right\| \leqslant a_{n}, \quad \lim _{n} a_{n}=0
$$

and $S=\Sigma_{j=1}^{\infty} X_{j}$ converges a.s., then for all $\epsilon>0, E[\exp (\epsilon\|S\|)]<\infty$.

Proof. As before, we may assume that the $X_{n}$ 's are symmetric. It is also clear that we may take $a_{n} \downarrow$. Let 


$$
M(k)=\sup _{n \geqslant 0}\left\|\sum_{j=k}^{k+n} X_{j}\right\|, \quad S(k)=\sum_{j=k}^{\infty} X_{j},
$$

then $M(k) \rightarrow 0$ a.s. as $k \rightarrow \infty$, and there exist positive numbers $t_{k} \rightarrow 0$ such that

$$
P\left[M(k)>t_{k}\right] \leqslant e^{-2} \leqslant P\left[M(k) \geqslant t_{k}\right] .
$$

Moreover, by Lemmas 2.1 and 3.4 we have for $t>0$

$$
\begin{aligned}
P[M(k)> & \left.2 t+a_{k}\right] \leqslant 2 P\left[\|S(k)\|>2 t+a_{k}\right] \\
& \leqslant 2 P\left[\sup _{j \geqslant k}\left\|X_{j}\right\|>a_{k}\right]+8 P^{2}[\|S(k)\|>t] \leqslant 8 P^{2}[M(k)>t] .
\end{aligned}
$$

From (3.38) and (3.39) it follows that there exist constants $A_{k}$ such that, for $t \geqslant t_{k}, P[M(k)>t] \leqslant A_{k} e^{-\epsilon} k^{t}$ where $\epsilon_{k}=\left(t_{k}+a_{k}\right)^{-1} \rightarrow \infty$ as $k \rightarrow \infty$. If $\epsilon>0$ is given, we may pick $k$ large so that $\epsilon_{k}>\epsilon$, and with this choice of $k$, observing that $\|S\| \leqslant \sum_{j=1}^{k-1} a_{j}+M(k)$ we have, for $t \geqslant t_{k}, P[\|S\|>t] \leqslant B_{k} e^{-\epsilon_{k} t}$ for some $B_{k}<\infty$. This clearly suffices to conclude that $E[\exp (\epsilon\|S\|)]<\infty$.

4. A special case. In this section we will examine the results of the previous section in a special situation. We let

$$
X_{n}=v_{n} Y_{n}, \quad n \geqslant 1,
$$

where $v_{n}$ are nonrandom elements of $B$ and the $Y_{n}$ are independent real-valued random variables. $S_{k}, M_{k}, S, M, N$ have the same meaning as before with $X_{n}=$ $v_{n} Y_{n}$. The questions that we are going to consider are of the following type: if the $Y_{n}$ are independent, $\sup _{n} E\left(\left|Y_{n}\right|^{p}\right)<\infty$ for some $p>0$, then does $M<\infty$ a.s. imply $E\left(M^{p}\right)<\infty$ or even $E\left(M^{q}\right)<\infty$ for $q<p$ ? The answer is that this is true only if additional conditions are imposed either on the distributions of $\left|Y_{n}\right|$ or on the sequence $\left\{\left\|v_{n}\right\|\right\}$. Otherwise, we will show by a counterexample that $M$ need not have any finite moment even though $Y_{n}$ does and $M<\infty$ a.s. The situation in the exponential case is somewhat different and is considered in Theorem 4.7.

The assumption that $M<\infty$ a.s. imposes a necessary condition on the distribution of $Y_{n}$ and on $\left\|v_{n}\right\|$. Since $M<\infty$ a.s. implies $N<\infty$ a.s., it follows from Remark 3.2 that there exists $u>0$ such that

$$
\sum_{n} P\left(\left\|v_{n}\right\|\left|Y_{n}\right|>u\right)<\infty .
$$

Using this observation we can show that if either the distribution of $\left|Y_{n}\right|$ or the sequence $\left\{\left\|v_{n}\right\|\right\}$ satisfies some regularity properties then moments of $M$ exist. One such result is the following:

THEOREM 4.1. Let $Y_{n}$ in (4.1) be independent and identically distributed 
random variables. Let $F(u)=P\left(\left|Y_{1}\right| \leqslant u\right)$ and $Q=1-F$. Assume that $Q$ is regularly varying and $E\left|Y_{1}\right|^{p}<\infty$ for some $p>0$. Suppose $M<\infty$ a.s. Then $E\left(M^{p-\epsilon}\right)<\infty, 0<\epsilon \leqslant p$.

Proof. This is essentially Theorem 6.1 in [3]. It also follows easily from Corollary 3.5 .

It is easy to see, by constructing a counterexample, that the theorem is false for $\epsilon=0$.

In the next theorem we will show that if $P\left(\left|Y_{1}\right|>u\right)$ does not decrease to zero too fast as $u \rightarrow \infty$, then the regularity of $\left\{\left\|v_{n}\right\|\right\}$ is enough to insure the existence of moments of $M$.

THEOREM 4.2. Let $Y_{n}$ in (4.1) be independent and identically distributed random variables. Assume that for some $p>0, E\left(\left|Y_{1}\right|^{p}\right)<\infty$ but $E\left(\left|Y_{1}\right|^{q}\right)=\infty$ for $q>p$. Let $\left\{\left\|v_{n}\right\|\right\}$ be regularly varying and $M<\infty$ a.s., then $E\left(M^{p-\epsilon}\right)<\infty$, $0<\epsilon \leqslant p$.

Proof. Without loss of generality we may assume $\left\|v_{n}\right\| \neq 0$ for all $n$. Furthermore, since rearrangement of terms will not affect the result we may assume $\left\{\left\|v_{n}\right\|\right\}$ is a nondecreasing sequence. Let $\alpha_{0}=0 ; \alpha_{j}=\left\|v_{j}\right\|^{-1}$ for $j \geqslant 1$. Since $M<\infty$ a.s. and $Y_{j}$ identically distributed, (4.2) allows us to conclude that $\alpha_{j}\left\lceil\infty\right.$. We write $F(u)=P\left(\left|Y_{1}\right| \leqslant u\right) ; Q=1-F$. The sequence $\left\{\alpha_{j}\right\}$ is regularly varying, hence

$$
\alpha_{j}=j^{\theta} l(j)
$$

for some $\theta \geqslant 0$ and $l$ a slowly varying function. By [1, Corollary, p. 274] we can find a regularly varying sequence $\left\{\beta_{j}\right\}$ such that

$$
\beta_{0}=0 ; \quad \beta_{j}=\gamma j^{\theta} \exp \left[\int_{1}^{j}(\epsilon(y) / y) d y\right], \quad j \geqslant 1
$$

and

$$
\lim _{j \rightarrow \infty} \beta_{j} / \alpha_{j}=1,
$$

for some $\gamma>0, \epsilon(y) \rightarrow 0$. For $a>0$, we have

$$
\begin{aligned}
E\left(\left|Y_{1}\right|^{q}\right) & =\sum_{j=0}^{\infty} \int_{\left(a \alpha_{j}, a \alpha_{j+1}\right)} x^{q} d F(x) \leqslant \sum_{j=0}^{\infty} a^{q} \alpha_{j+1}^{q}\left[Q\left(a \alpha_{j}\right)-Q\left(a \alpha_{j+1}\right)\right] \\
& \leqslant c_{1} a^{q} \sum_{j=0}^{\infty} \beta_{j+1}^{q}\left[Q\left(a \alpha_{j}\right)-Q\left(a \alpha_{j+1}\right)\right] \\
& \leqslant c_{1} a^{q} \sum_{j=0}^{\infty} Q\left(a \alpha_{j}\right)\left[\beta_{j+1}^{q}-\beta_{j}^{q}\right] \leqslant c_{2} a^{q} \sum_{j=0}^{\infty} Q\left(a \alpha_{j}\right) j^{\theta q-1} l^{q}(j),
\end{aligned}
$$

for some positive constants $c_{1}$ and $c_{2}$, by using the representation in (4.4) for 
$\beta_{j}$. By (4.2) we have for some $a>0$

$$
\sum_{j} Q\left(a \alpha_{j}\right)<\infty .
$$

Since $E\left(\left|Y_{1}\right|^{q}\right)=\infty$ for $q>p,(4.6)$ and (4.7) imply that $\theta \geqslant p^{-1}$. If $\theta>p^{-1}$, then $\Sigma_{j}\left\|v_{j}\right\|^{p}=\Sigma_{j} j^{-\theta p} l^{-1}(j)<\infty$ and consequently by Corollary 3.5 we have $E\left(M^{p}\right)<\infty$. Therefore assume $\theta=p^{-1}$. We now proceed to check condition

(c) of Corollary 3.5 with $p-\epsilon$ in place of $p$. In order to check this note that we already have $\Sigma Q\left(a \alpha_{j}\right)<\infty$ for some $a>0$ by (4.7). Also

$$
\begin{aligned}
\left\|v_{n}\right\|^{p-\epsilon} \int_{a\left\|v_{n}\right\|^{-1}}^{\infty} Q(u) d u^{p-\epsilon} & \leqslant n^{-1+\epsilon \theta} l^{-p+\epsilon}(n) \sum_{j=n}^{\infty} \int_{a_{1} \beta_{j}}^{a_{1} \beta_{j+1}} Q(u) d u^{p-\epsilon} \\
& \leqslant c_{3} n^{-1+\epsilon \theta} l^{-p+\epsilon}(n) \sum_{j=n}^{\infty} Q\left(a_{2} \alpha_{j}\right) l^{p-\epsilon}(j) j^{-\epsilon \theta}
\end{aligned}
$$

for some positive constants $a_{1}, a_{2}$ and $c_{3}$. Now

$$
\begin{aligned}
& \sum_{n=1}^{\infty} n^{-1+\epsilon \theta} l^{-p+\epsilon}(n) \sum_{j=n}^{\infty} Q\left(a_{2} \alpha_{j}\right) l^{p-\epsilon}(j) j^{-\epsilon \theta} \\
& \quad=\sum_{j=1}^{\infty}\left(\sum_{n=1}^{j} n^{-1+\epsilon \theta} l^{-p+\epsilon}(n)\right) Q\left(a_{2} \alpha_{j}\right) l^{p-\epsilon}(j) j^{-\epsilon \theta} \leqslant c_{4} \sum_{j=1}^{\infty} Q\left(a_{2} \alpha_{j}\right)<\infty
\end{aligned}
$$

by using [1, Theorem 1, p. 273] and (4.7). Hence condition (c) of Corollary 3.5 is satisfied and the proof is complete.

We will now give a counterexample that shows that even though the $Y_{n}$ 's are independent identically distributed, $E\left(Y_{1}^{2}\right)<\infty$, and $M<\infty$ a.s. (indeed $\Sigma_{n} v_{n} Y_{n}$ converges a.s.), $E\left(M^{\epsilon}\right)=\infty$ for every $\epsilon>0$.

EXAMPLE 4.3. We take $B=C[0,1]$, the space of continuous functions on $[0,1]$ with the sup norm. For $\varphi \in C[0,1]$, we will denote its sup norm by $\|\varphi\|$. For $j \geqslant 0$, let $\varphi_{j} \geqslant 0$ be given by

$$
\varphi_{j}^{2}(t)=\left\{\begin{array}{l}
0 \text { for } t \notin\left(2^{-j-1}, 2^{-j}\right), \\
2^{j+2} \text { for } t=3 \cdot 2^{-j-2} \\
\text { linear in between }
\end{array}\right.
$$

Thus the $\varphi_{j}$ form an orthonormal system in $L^{2}[0,1]$. Define

$$
v_{j}=\lambda_{j} \varphi_{j},
$$

where the $\lambda_{j}$ are positive real numbers and will be chosen suitably later. Let $Y_{j}, j \geqslant 1$, be independent identically distributed random variables on some $(\Omega, F, P)$, each with distribution $F$. We will pick $F$ later so $E\left(Y_{1}^{2}\right)<\infty$. Let

$$
Q(x)=P\left(\left|Y_{1}\right|>x\right)
$$

and define 


$$
X(t)=\sum_{j=0}^{\infty} v_{j}(t) Y_{j}
$$

The following lemma gives necessary and sufficient conditions for the uniform convergence of the series in (4.10).

Lemma 4.4. Let $v_{j}, Y_{j}$ and $Q$ be as above. Then the series in (4.10) converges uniformly if and only if

$$
\sum_{j=0}^{\infty} Q\left(a /\left\|v_{j}\right\|\right)<\infty, \quad \forall a>0 .
$$

Proof. Since the $v_{j}$ have disjoint supports, the series in (4.10) converges uniformly a.s. if and only if for almost all $\omega, \forall a>0, \exists n_{0}(a, \omega)$ such that

$$
\sup _{n \geqslant n_{0}}\left(\left\|v_{j}\right\|\left|Y_{j}\right|\right) \leqslant a .
$$

By the Borel-Cantelli lemma, (4.11) holds if and only if (4.12) holds. Hence the proof.

We now proceed with our example. We write

$$
\gamma_{j}=\left\|v_{j}\right\|^{-1} \text {. }
$$

For $m \geqslant 1$ let $u_{m}=2^{m(m-1) / 2}$, and pick $\gamma_{j}>0$ so that

$$
\gamma_{j}=u_{m} \quad \text { for } u_{0}^{2}+\cdots+u_{m}^{2} \leqslant j<u_{0}^{2}+\cdots+u_{m+1}^{2} \text {. }
$$

Also define

$$
Q(x)=\left\{\begin{array}{l}
\left(m^{2} u_{m+1}^{2}\right)^{-1} \text { for } 2^{-(m-1) / 2} u_{m}<x \leqslant 2^{-m / 2} u_{m+1}, \\
1 \text { for } x \in[0,1] .
\end{array}\right.
$$

We first check that $E\left(Y_{1}^{2}\right)<\infty$, which follows from

$$
\int_{0}^{\infty} x Q(x) d x \leqslant 1+\sum_{m=1}^{\infty}\left(m^{2} u_{m+1}^{2}\right)^{-1}\left(2^{-m / 2} u_{m+1}\right)^{2}<\infty .
$$

We also check (4.11) which is equivalent to checking

$$
\sum_{j} Q\left(a \gamma_{j}\right)<\infty
$$

for all $a>0$. From (4.14) we get for $0<a \leqslant 1$

$$
\sum_{j=1}^{\infty} Q\left(a \gamma_{j}\right)=\sum_{m=1}^{\infty} 2^{m(m+1)} Q\left(a u_{m}\right)
$$

Given $0<a \leqslant 1$, there exists $m_{0}$ such that for $m \geqslant m_{0}$

$$
2^{-(m-1) / 2} u_{m} \leqslant a u_{m} \leqslant 2^{-m / 2} u_{m+1},
$$

hence 


$$
\sum_{m=m_{0}}^{\infty} 2^{m(m+1)} Q\left(a u_{m}\right) \leqslant \sum_{m=m_{0}}^{\infty} 2^{m(m+1)}\left(m^{2} u_{m+1}^{2}\right)^{-1}<\infty
$$

and (4.16) is checked and by Lemma 4.4 the series in (4.10) converges uniformly a.s. We will now show that $E\left(\|S\|^{\epsilon}\right)=\infty$ for all $\epsilon>0$, where $S=\Sigma v_{j} Y_{j}$. By Corollary 3.5 we have

$$
E\left(\|S\|^{\epsilon}\right)<\infty \Leftrightarrow \sum_{n=1}^{\infty} E\left(\left\|v_{n}\right\|^{\epsilon}\left|Y_{n}\right|^{\epsilon} I\left[\left|Y_{n}\right|>a /\left\|v_{n}\right\|\right]\right)<\infty
$$

for some $a>0$. We will show that the sum on the right side diverges for all $a>0$. Now

$$
\begin{gathered}
E\left(\left\|v_{n}\right\|^{\epsilon}\left|Y_{n}\right|^{\epsilon} I\left[\left|Y_{n}\right|>a /\left\|v_{n}\right\|\right]\right)=\left\|v_{n}\right\|^{\epsilon} \int_{\left(a /\left\|v_{n}\right\|, \infty\right)} u^{\epsilon} d P\left(\left|Y_{1}\right| \leqslant u\right) \\
\geqslant \epsilon\left\|v_{n}\right\|^{\epsilon} \int_{a /\left\|v_{n}\right\|}^{\infty} Q(u) u^{\epsilon-1} d u=\epsilon \int_{a}^{\infty} Q\left(u /\left\|v_{n}\right\|\right) u^{\epsilon-1} d u .
\end{gathered}
$$

Hence recalling (4.13), (4.14) and (4.15) we see that the sum in (4.18) dominates

$$
\begin{aligned}
\epsilon \sum_{n=1}^{\infty} \int_{a}^{\infty} Q\left(x \gamma_{n}\right) x^{\epsilon-1} d x & =\epsilon \sum_{m=1}^{\infty} 2^{m(m+1)} \int_{a}^{\infty} Q\left(x u_{m}\right) x^{\epsilon-1} d x \\
& \geqslant \epsilon \sum_{m=n}^{\infty} 2^{m(m+1)} \int_{a}^{2^{(m-1) / 2}} Q\left(x u_{m}\right) x^{\epsilon-1} d x \\
& \geqslant \sum_{m=n}^{\infty} 2^{m(m+1)} Q\left(2^{(m-1) / 2} u_{m}\right)\left(2^{\epsilon(m-1) / 2}-a^{\epsilon}\right) \\
& =\sum_{m=n}^{\infty} 2^{m(m+1)}\left(m^{2} u_{m+1}^{2}\right)^{-1}\left(2^{\epsilon(m-1) / 2}-a^{\epsilon}\right),
\end{aligned}
$$

where $n$ is chosen large enough so $2^{(n-1) / 2} \geqslant a$ and equality at the last step follows by observing that $2^{-(m-1) / 2} u_{m} \leqslant 2^{(m-1) / 2} u_{m} \leqslant 2^{-m / 2} u_{m+1}$. The last sum clearly diverges and the demonstration of the counterexample is complete.

Remark 4.5. By a similar argument, for any $p>0$, one can choose the $Y_{n}$ 's such that $E\left(\left|Y_{1}\right|^{p}\right)<\infty, M<\infty$ a.s. and $E\left(M^{\epsilon}\right)=\infty$ for every $\epsilon>0$.

We now consider the exponential case in the following theorem.

THEOREM 4.6. Let $X_{n}$ be as in (4.1). Suppose $M<\infty$ a.s. If for some $\alpha>0, \beta>0, c>0$, and all $u \geqslant 1$

$$
\begin{aligned}
& P\left(\left|Y_{n}\right|>u\right) \leqslant \exp \left(-\alpha u \log ^{1+c} u\right) \\
& \sum_{n=1}^{\infty} \exp \left(-\frac{\beta}{\left\|v_{n}\right\|} \log ^{1+c} \frac{1}{\left\|v_{n}\right\|}\right)<\infty,
\end{aligned}
$$

then there exists $\epsilon>0$ such that $E(\exp (\epsilon M))<\infty$.

Proof. First observe that $M .<\infty$ a.s. implies $N=\sup _{n}\left\|X_{n}\right\|<\infty$ a.s. 
Hence by Remark 3.2 there exists $a>0$ such that

$$
\sum_{n} P\left(\left|Y_{n}\right|>\frac{a}{\left\|v_{n}\right\|}\right)<\infty .
$$

Let $F_{n}(u)=P\left(X_{n} \leqslant u\right), Q_{n}=1-F_{n}$. We apply Theorem 3.8 . Hence we look at $(\delta>0, b>0)$

$$
\begin{aligned}
\sum_{n=1}^{\infty} \int_{[b, \infty)} \exp (\delta & \left.u \log ^{1+c} u\right) d F_{n}(u) \\
& =\sum_{n=1}^{\infty}\left\{Q_{n}(b) \exp \left(\delta b \log ^{1+c} b\right)+\int_{b}^{\infty} Q_{n}(u) d\left(e^{\delta u \log 1+c} u\right)\right\}
\end{aligned}
$$

and for $b \geqslant \max (a, 1)$, using (4.19) and (4.20)

$$
\leqslant A+B \sum_{n=1}^{\infty} \int_{b}^{\infty} \exp \left(-\frac{\alpha u}{\left\|v_{n}\right\|} \log ^{1+c} \frac{u}{\left\|v_{n}\right\|}\right) \exp \left(2 \delta u \log ^{1+c} u\right) d u
$$

where $A, B$ are finite constants. By (4.19) there is a $\gamma>0$ such that $\left\|v_{n}\right\| \leqslant \gamma$, hence we can dominate this last expression by

$$
A+B \sum_{n=1}^{\infty} \int_{b}^{\infty} \exp \left(-\frac{\theta u}{\left\|v_{n}\right\|} \log ^{1+c} \frac{u}{\left\|v_{n}\right\|}\right) d u,
$$

for some $\theta>0$ by picking $\delta$ sufficiently small. Since $b$ can be chosen as large as we wish, the convergence of

$$
\sum_{n=1}^{\infty} \exp \left(-\frac{\beta}{\left\|v_{n}\right\|} \log ^{1+c} \frac{1}{\left\|v_{n}\right\|}\right)
$$

implies that of the expression in (4.21). Hence by Theorem 3.8 the result follows.

If the $Y_{n}$ are uniformly bounded random variables, then we have the following immediate consequence of Theorem 3.8.

THEOREM 4.7. Let $X_{n}$ be as in (4.1). Suppose that $\left|Y_{n}\right| \leqslant A<\infty$ a.s. for all $n$, and that either

(a) $\left\|v_{n}\right\| \leqslant B<\infty$ for all $n$, or

(b) $Y_{n}$ are identically distributed.

Then $M<\infty$ a.s. implies that for some $\epsilon>0$

$$
E(\exp (\epsilon M))<\infty \text {. }
$$

Proof. $M<\infty$ a.s. implies $\sup _{n}\left(\left\|v_{n}\right\|\left|Y_{n}\right|\right)<\infty$ a.s., hence under (b) we must have $\sup _{n}\left\|v_{n}\right\|<B$ for some $B<\infty$. Hence (b) implies (a) and it is enough to prove the result under (a). If (a) holds, then $\left\|X_{n}\right\|=\left\|v_{n}\right\|\left|Y_{n}\right| \leqslant A B$ a.s. for all $n$. Hence (4.22) follows from Theorem 3.8 by taking $a>A B$ in (3.25). This completes the proof.

Theorem 4.7 is proved in [5, Theorem 4, p. 27] when $\left\{Y_{n}\right\}$ is a Rademacher sequence. Under the added condition that $\Sigma X_{n}$ converges to $S,(4.22)$ is also 
obtained with $\|S\|$ replacing $M$. In the next theorem, which is a special case of Theorem 3.11, we have this result holding for all $\epsilon>0$.

THEOREM 4.8. Let $\left\{X_{n}\right\}$ be as in (4.1). Suppose the $Y_{n}$ are independent identically distributed and $\sup _{n}\left|Y_{n}\right| \leqslant A<\infty$ a.s. If $\Sigma_{n=1}^{\infty} v_{n} Y_{n}$ converges to $S$, then

$$
\forall \epsilon>0, \quad \dot{E(\exp (\epsilon\|S\|))<\infty .}
$$

REMARK 4.9. If $B=R^{1},\left\{Y_{i}\right\}$ a Rademacher sequence, then it is known that $E\left(\exp \left(\epsilon\|S\|^{2}\right)\right)<\infty$ for all $\epsilon>0$. It is likely that this is true when $B$ is any separable Banach space. We will obtain this stronger result in $\S 6$ under additional conditions. In fact, this result will apply to any sequence of independent uniformly bounded, symmetric random variables $\left\{Y_{i}\right\}$.

5. Comparison theorems for sums of independent vector-valued random variables. Let $\left\{u_{n}\right\}$ be a sequence of elements in $B$ and $\left\{\epsilon_{n}\right\}$ a Rademacher sequence of random variables on some probability space $(\Omega, F, P)$, i.e. the $\epsilon_{n}$ are independent, identically distributed random variables such that $P\left[\epsilon_{1}=1\right]=P\left[\epsilon_{1}=-1\right]$ $=1 / 2$. Kahane $[5$, Theorem 5, p. 18] gives the following very useful result which he calls a contraction principle: If the random series $\sum_{n=1}^{\infty} \epsilon_{n} u_{n}$ converges in norm a.s. (or is bounded) and $\lambda_{n}$ is a bounded scalar sequence, then the random series $\sum_{n=1}^{\infty} \epsilon_{n} \lambda_{n} u_{n}$ converges in norm a.s. (or is bounded). Our aim here is to generalize this contraction principle in several directions. The nature of the generalizations leads us to call our results comparison theorems.

Let $\left\{\eta_{n}, n \geqslant 1\right\}$ be a sequence of independent, real-valued, random variables on a probability space $(\Omega, F, P)$ and $\left\{u_{n}, n \geqslant 1\right\}$ be a sequence of elements in $B$. We will be concerned with the following questions: If the series $\Sigma_{k=1}^{\infty} \eta_{k} u_{k}$ is a.s. convergent (or a.s. bounded), and if $\left\{\xi_{k}\right\}$ is some other sequence of independent, real-valued random variables (possibly on a different probability space) such that $\xi_{k}$ is "smaller" than $\eta_{k}$, in some sense, for all $k \geqslant 1$, then is the series $\sum_{k=1}^{\infty} \xi_{k} u_{k}$ a.s. convergent (or a.s. bounded)? Also, if $\varphi$ is a nondecreasing, nonnegative convex function defined on $[0, \infty)$, and

$$
E\left[\varphi\left(\sup _{n}\left\|\sum_{k=1}^{n} \eta_{k} u_{k}\right\|\right)\right]<\infty
$$

or if the series converges a.s., and

$$
E\left[\varphi\left(\left\|\sum_{k=1}^{\infty} \eta_{k} u_{k}\right\|\right)\right]<\infty,
$$

then do (5.1) and (5.2) remain valid if the $\eta_{k}$ 's are replaced by the "smaller" $\xi_{k}$ 's?

Our results include the above mentioned result of Kahane and its generaliza- 
tion by Hoffmann-J $\phi$ rgensen [3]. Many of their ideas are used in our proofs. We also use a technique of Pisier [8]. Before stating the main results we introduce some notation.

If the random variables of the sequence $\left\{\eta_{k}, k \geqslant 1\right\}$ are symmetric, then they are said to be uniformly nondegenerate if there exist $a, b>0$ such that

$$
P\left[\left|\eta_{k}\right| \geqslant a\right] \geqslant b, \quad \forall k \geqslant 1 .
$$

In general the $\eta_{k}$ are called uniformly nondegenerate if there exist $c, d>0$ such that

$$
P\left[\left|\eta_{k}-\eta_{k}^{\prime}\right| \geqslant c\right] \geqslant d, \quad \forall k \geqslant 1,
$$

where $\left\{\eta_{k}^{\prime}\right\}$ is an independent copy of the sequence $\left\{\eta_{k}\right\}$. Clearly (5.3) can be included in (5.4) but it will be convenient to have both definitions.

REMARK 5.0. All our results that will be stated below involve two sequences of independent random variables $\left\{\eta_{k}\right\}$ and $\left\{\xi_{k}\right\}$ which could be given on different probability spaces. Since the results depend only on the finite-dimensional distributions of the $\eta_{k}$ 's, and the finite-dimensional distributions of the $\xi_{k}$ 's, and not on the structure of the underlying probability space(s), we can assume that both sequences are defined on the same probability space. (We can simply take the two sequences independent of each other.) We will often need to introduce a Rademacher sequence which is independent of $\left\{\eta_{k}\right\}$ and $\left\{\xi_{k}\right\}$. Hence even if the two sequences $\left\{\eta_{k}\right\}$ and $\left\{\xi_{k}\right\}$ were originally given on different probability spaces, without any loss of generality we will assume that there is a basic probability space $(\Omega, F, P)$ $=\left(\Omega_{1} \times \Omega_{2}, F_{1} \times F_{2}, P_{1} \times P_{2}\right)$ such that the sequences $\left\{\eta_{k}\right\}$ and $\left\{\xi_{k}\right\}$ are given on $\left(\Omega_{1}, F_{1}, P_{1}\right)$ and an independent Rademacher sequence $\left\{\epsilon_{n}\right\}$ on $\left(\Omega_{2}, F_{2}, P_{2}\right)$. They are then defined on the product space in the usual manner. $E_{1}, E_{2}$ and $E$ will denote expectation operators with respect to $P_{1}, P_{2}$ and $P$. On occasion we will choose $\left(\Omega_{1}, F_{1}, P_{1}\right)$ and $\left(\Omega_{2}, F_{2}, P_{2}\right)$ in some suitable manner.

Our first results are stated for symmetric $\eta_{k}$ 's. Remark 5.0 applies to each result.

THEOREM 5.1. Let $\left\{u_{k}, k \geqslant 1\right\}$ be a sequence of elements of $B$ and $\left\{\eta_{k}\right\}$ independent, symmetric, real-valued random variables on the probability space $(\Omega, F, P)$. Assume that the $\eta_{k}$ 's are uniformly nondegenerate. Let $\left\{\xi_{k}, k \geqslant 1\right\}$ be independent, symmetric, real-valued random variables on $(\Omega, F, P)$ such that, for some $x_{0} \geqslant 0,0<\alpha \leqslant 1$,

$$
P\left[\left|\eta_{k}\right| \geqslant x\right] \geqslant \alpha P\left[\left|\xi_{k}\right| \geqslant x\right],
$$

for all $x \geqslant x_{0}$. Then

$$
\sup _{n}\left\|\sum_{k=1}^{n} \eta_{k} u_{k}\right\|<\infty \text { a.s. } \Rightarrow \sup _{n}\left\|\sum_{k=1}^{n} \xi_{k} u_{k}\right\|<\infty \text { a.s. }
$$

and 


$$
\sum_{k=1}^{\infty} \eta_{k} u_{k} \text { converges a.s. } \Rightarrow \sum_{k=1}^{\infty} \xi_{k} u_{k} \text { converges a.s. }
$$

If in addition the $\xi_{k}$ are uniformly bounded, then (5.5) is satisfied, and we get the ímmediate

Corollary 5.2. Let $\left\{u_{k}\right\}$ and $\left\{\eta_{k}\right\}$ be as in Theorem 5.1. If $\left\{\xi_{k}\right\}$ is a sequence of independent, symmetric, real-valued, uniformly bounded random variables on $(\Omega, F, P)$, then (5.6) and (5.7) hold.

THEOREM 5.3. Let $\varphi$ be a nonnegative, nondecreasing, convex function on $[0, \infty)$. Let $\left\{u_{k}\right\},\left\{\eta_{k}\right\},\left\{\xi_{k}\right\}$ be as in Theorem 5.1. Suppose (5.5) holds with $x_{0}=0$. Then

$$
E\left[\varphi\left(\sup _{n}\left\|\sum_{k=1}^{n} \xi_{k} u_{k}\right\|\right)\right] \leqslant E\left[\varphi\left(\alpha_{n}^{-1} \sup _{n}\left\|\sum_{k=1}^{n} \eta_{k} u_{k}\right\|\right)\right] ;
$$

if $\Sigma_{k=1}^{\infty} \eta_{k} u_{k}$ converges a.s., so does $\Sigma_{k=1}^{\infty} \xi_{k} u_{k}$, and in this case (5.8) holds with $\left\|\Sigma_{k=1}^{\infty}\right\|$ replacing $\sup _{n}\left\|\Sigma_{k=1}^{n}\right\|$.

If $x_{0}>0$ in (5.5), let $a, b$ be any values for which (5.j) holds. Then

$$
E\left[\varphi\left(\sup _{n}\left\|\sum_{k=1}^{n} \xi_{k} u_{k}\right\|\right)\right]
$$

$$
\leqslant \frac{1}{2} E\left[\varphi\left(\frac{2 x_{0}}{\alpha a b} \sup _{n}\left\|\sum_{k=1}^{n} \eta_{k} u_{k}\right\|\right)\right]+\frac{1}{2} E\left[\varphi\left(\frac{2}{\alpha} \sup _{n}\left\|\sum_{k=1}^{n} \eta_{k} u_{k}\right\|\right)\right] \text {. }
$$

If $\sum_{k=1}^{\infty} \eta_{k} u_{k}$ converges a.s. so does $\sum_{k=1}^{\infty} \xi_{k} u_{k}$ and (5.9) holds with $\left\|\Sigma_{k=1}^{\infty}\right\|$ replacing $\sup _{n}\left\|\Sigma_{k=1}^{n}\right\|$.

All the proofs will be given at the end of this section.

The symmetry of $\left\{\xi_{k}\right\}$ is essential to our method of proof; however, in most cases we can remove the symmetry assumption on $\left\{\eta_{k}\right\}$. That this cannot always be done will be shown by examples later in this section.

In the general case, when the $\eta_{k}$ need not be symmetric, we will need another uniform condition, that there exist a $\gamma>0$ and a $\delta>0$ such that

$$
P\left[\left|\eta_{k}\right|<\gamma\right] \geqslant \delta, \quad \forall k \geqslant 1 .
$$

We then obtain

THEOREM 5.4. Let $\left\{u_{k}\right\}$ be a sequence of elements of $B$, and $\left\{\eta_{k}\right\}$ a sequence of independent, real-valued random variables on $(\Omega, F, P)$ that are uniformly nondegenerate. Also assume that $\left\{\eta_{k}\right\}$ satisfies (5.10) for some $\gamma>0$ and $\delta>0$. Let $\left\{\xi_{k}\right\}$ be independent, symmetric, real-valued, random variables on $(\Omega, F, P)$ such that, for some $x_{0} \geqslant 0,0<\alpha \leqslant 1$, 


$$
P\left[\left|\eta_{k}\right| \geqslant x\right] \geqslant \alpha P\left[\left|\xi_{k}\right| \geqslant x\right]
$$

for all $x \geqslant x_{0}$. Then

$$
\sup _{n}\left\|\sum_{k=1}^{n} \eta_{k} u_{k}\right\|<\infty \text { a.s. } \Rightarrow \sup _{n}\left\|\sum_{k=1}^{n} \xi_{k} u_{k}\right\|<\infty \text { a.s. }
$$

and

$$
\sum_{k=1}^{\infty} \eta_{k} u_{k} \text { converges a.s. } \Rightarrow \sum_{k=1}^{\infty} \xi_{k} u_{k} \text { converges a.s. }
$$

REMARK 5.5'. If we take $\xi_{k}=\epsilon_{k} \eta_{k}$, where $\left\{\epsilon_{k}\right\}$ is a Rademacher sequence in $(\Omega, F, P)$ independent of $\left\{\eta_{k}\right\}$, then Theorem 5.4 shows that if the $\eta_{k}$ satisfy (5.4) and (5.10), then $\Sigma_{k=1}^{\infty} \eta_{k} u_{k}$ converges a.s. (or is bounded a.s.) $\Rightarrow \sum_{k=1}^{\infty} \epsilon_{k} \eta_{k} u_{k}$ converges a.s. (or is bounded a.s.).

For use in an analogue of Theorem 5.3 we define

(5.14) $v_{0}=\max \left(x_{0}, 2 \gamma\right)$, where $x_{0}$ appears following (5.11) and $\gamma$ in (5.10).

THEOREM 5.5. Let $\varphi$ be a nonnegative, nondecreasing, convex function on $[0, \infty)$. Let $\left\{u_{k}\right\},\left\{\eta_{k}\right\}$ and $\left\{\xi_{k}\right\}$ be defined as in Theorem 5.4. Then for $c, d$ any values for which (5.4) holds, we have

$$
\begin{aligned}
& 2 E\left[\varphi\left(\sup _{n}\left\|\sum_{k=1}^{n} \xi_{k} u_{k}\right\|\right)\right] \\
& \leqslant E\left[\varphi\left(c_{1} \sup _{n}\left\|\sum_{k=1}^{n} \eta_{k} u_{k}\right\|\right)\right]+E\left[\varphi\left(c_{2} \sup _{n}\left\|\sum_{k=1}^{n} \eta_{k} u_{k}\right\|\right)\right],
\end{aligned}
$$

where $c_{1}=4 v_{0} / \alpha c d \delta$ and $c_{2}=8 / \alpha \delta$, with $v_{0}$ given in (5.14) and $\gamma, \delta$ as in (5.10).

Furthermore, if $\Sigma_{k=1}^{\infty} \eta_{k} u_{k}$ converges a.s., then so does $\Sigma_{k=1}^{\infty} \xi_{k} u_{k}$ and (5.15) holds with $\left\|\Sigma_{k=1}^{\infty}\right\|$ replacing $\sup _{n}\left\|\sum_{k=1}^{n}\right\|$.

There is also an anlogue of Corollary 5.2, the proof of which follows by symmetrization from (5.4) and Theorem 5.1.

THEOREM 5.6. Let $\left\{u_{k}\right\}$ be a sequence of elements in $B$, and $\left\{\eta_{k}\right\}$ be independent, real-valued random variables on $(\Omega, F, P)$ that are uniformly nondegenerate. Let $\left\{\xi_{k}\right\}$ be independent, symmetric, real-valued random variables on $(\Omega, F, P)$ that are uniformly bounded. Then (5.12) and (5.13) hold.

We will now give some examples to show why (5.4) and (5.10) cannot be dropped in Theorem 5.4.

In the first example the $\eta_{k}$ are nondegenerate, but not uniformly nondegenerate. ((5.4) does not hold.) Let $B=R^{1}$ and

$$
\begin{aligned}
& u_{k}=0 \text { for } k=1,2,3, \\
& u_{2 k}=(\log k)^{-1}, k \geqslant 2, \\
& u_{2 k+1}=(-\log k)^{-1}, k \geqslant 2 .
\end{aligned}
$$


Let $\left\{\eta_{k}\right\}$ be independent random variables with

$$
P\left[\eta_{k}=1+2^{-k}\right]=P\left[\eta_{k}=1-2^{-k}\right]=1 / 2 .
$$

Then $\Sigma_{k=1}^{\infty} \eta_{k} u_{k}$ converges a.s., but $\sum_{k=1}^{\infty} \epsilon_{k} \eta_{k} u_{k}$ does not converge a.s. if $\left\{\epsilon_{k}\right\}$ is a Rademacher sequence independent of $\left\{\eta_{k}\right\}$. Hence by Remark 5.5', Theorem 5.4 cannot hold.

In the next example $\left\{\eta_{k}^{\prime}\right\}$ is uniformly nondegenerate but (5.10) does not hold. Let $\left\{\eta_{k}\right\}$ and $\left\{u_{k}\right\}$ be as above, and $\eta_{k}^{\prime}=2^{k} \eta_{k}, u_{k}^{\prime}=u_{k} 2^{-k}$, then $\Sigma_{k=1}^{\infty} \eta_{k}^{\prime} u_{k}^{\prime}$ converges a.s., but $\Sigma_{k=1}^{\infty} \epsilon_{k} \eta_{k}^{\prime} u_{k}^{\prime}$ again does not converge a.s. and Theorem 5.4 cannot hold.

The essential role of (5.3) in Theorem 5.1 can be demonstrated in a similar manner.

We begin the proofs with a series of lemmas. The first lemma is an extension of [3, Lemma 4.1] (see remark below); our method of proof follows Kahane $[5$, p. 18].

LEMMA 5.7. Let $\varphi$ be a nonnegative, nondecreasing, convex function defined on $[0, \infty)$ and $\lambda_{1}, \lambda_{2}, \ldots$ real constants such that $\sup _{k}\left|\lambda_{k}\right| \leqslant c$. Let $\left\{u_{k}\right\}$ be a sequence of elements in $B$ and $\left\{\eta_{k}\right\}$ independent, symmetric, real-valued random variables on $(\Omega, F, P)$. Then

$$
E\left[\varphi\left(\left\|\sum_{k=1}^{n} \lambda_{k} \eta_{k} u_{k}\right\|\right)\right] \leqslant E\left[\varphi\left(c\left\|\sum_{k=1}^{n} \eta_{k} u_{k}\right\|\right)\right]
$$

for each $n$, and

$$
E\left[\varphi\left(\sup _{n}\left\|\sum_{k=1}^{n} \lambda_{k} \eta_{k} u_{k}\right\|\right)\right] \leqslant E\left[\varphi\left(c \sup _{n}\left\|\sum_{k=1}^{n} \eta_{k} u_{k}\right\|\right)\right]
$$

Proof. We will prove (5.17); the proof of (5.16) is similar. Case (i). $\lambda_{k}=0$ or 1 . In this case

$$
\sum_{k=1}^{n} \lambda_{k} \eta_{k} u_{k}=\frac{1}{2}\left(\sum_{k=1}^{n}\left(2 \lambda_{k}-1\right) \eta_{k} u_{k}+\sum_{k=1}^{n} \eta_{k} u_{k}\right)
$$

Therefore

$$
\sup _{n}\left\|\sum_{k=1}^{n} \lambda_{k} \eta_{k} u_{k}\right\| \leqslant \frac{1}{2}\left(\sup _{n}\left\|\sum_{k=1}^{n}\left(2 \lambda_{k}-1\right) \eta_{k} u_{k}\right\|+\sup _{n}\left\|\sum_{k=1}^{n} \eta_{k} u_{k}\right\|\right)
$$

Since $\varphi$ is convex and nondecreasing, we get

$$
\varphi\left(\sup _{n}\left\|\sum_{k=1}^{n} \lambda_{k} \eta_{k} u_{k}\right\|\right) \leqslant \frac{1}{2}\left[\varphi\left(\sup _{n}\left\|\sum_{k=1}^{n}\left(2 \lambda_{k}-1\right) \eta_{k} u_{k}\right\|\right)+\varphi\left(\sup _{n}\left\|\sum_{k=1}^{n} \eta_{k} u_{k}\right\|\right)\right]
$$

Since the $\eta_{k}$ are symmetric $\Sigma_{k=1}^{n}\left(2 \lambda_{k}-1\right) \eta_{k} u_{k}$ and $\Sigma_{k=1}^{n} \eta_{k} u_{k}$ are stochastically 
similar, consequently

$$
E\left[\varphi\left(\sup _{n}\left\|\sum_{k=1}^{n} \lambda_{k} \eta_{k} u_{k}\right\|\right)\right] \leqslant E\left[\varphi\left(\sup _{n}\left\|\sum_{k=1}^{n} \eta_{k} u_{k}\right\|\right)\right] .
$$

Case (ii). $0 \leqslant \lambda_{k} \leqslant 1$. (Since the $\eta_{k}$ are symmetric we may take $\lambda_{k} \geqslant 0$, $c$ can clearly be taken equal to 1 without any loss of generality.) Writing $\lambda_{k}$ in binary expansion

$$
\lambda_{k}=\sum_{i=1}^{\infty} 2^{-i} \lambda_{k i}, \quad \lambda_{k i}=1 \text { or } 0
$$

we have

$$
\sum_{k=1}^{n} \lambda_{k} \eta_{k} u_{k}=\sum_{k=1}^{n}\left(\sum_{i=1}^{\infty} 2^{-i} \lambda_{k i}\right) \eta_{k} u_{k}=\sum_{i=1}^{\infty} 2^{-i}\left(\sum_{k=1}^{n} \lambda_{k i} \eta_{k} u_{k}\right) .
$$

Again, since $\varphi$ is convex and nondecreasing,

$$
\varphi\left(\sup _{n}\left\|\sum_{k=1}^{n} \lambda_{k} \eta_{k} u_{k}\right\|\right) \leqslant \sum_{i=1}^{\infty} 2^{-i} \varphi\left(\sup _{n}\left\|\sum_{k=1}^{n} \lambda_{k i} \eta_{k} u_{k}\right\|\right) .
$$

Now taking expectations and using (5.18) we obtain (5.17) and the proof is complete.

REMARK 5.8. Lemma 5.7 is also valid with $\left\{\eta_{k} u_{k}\right\}$ replaced by $\left\{X_{k}\right\}$, where $X_{k}$ are independent symmetric $B$-valued random variables.

LEMMA 5.9. Let $\left\{u_{k}\right\}$ be a sequence of elements in $B$ and $\left\{\eta_{k}\right\}$ independent, symmetric, real-valued random variables on $(\Omega, F, P)$ that are uniformly nondegenerate and uniformly bounded. Assume that

$$
M=\sup _{n}\left\|\sum_{k=1}^{n} \eta_{k} u_{k}\right\|<\infty \text { a.s. }
$$

Then for any sequence $\left\{\xi_{k}\right\}$ of independent, symmetric, real-valued random variables on $(\Omega, F, P)$ that are uniformly bounded we have

$$
M^{\prime}=\sup _{n}\left\|\sum_{k=1}^{n} \xi_{k} u_{k}\right\|<\infty \text { a.s., }
$$

and $E(M)<\infty, E\left(M^{\prime}\right)<\infty$.

Furthermore, if $\Sigma_{k=1}^{\infty} \eta_{k} u_{k}$ converges a.s., then so does $\Sigma_{k=1}^{\infty} \xi_{k} u_{k}$ and both $\left\|\Sigma_{k=1}^{\infty} \eta_{k} u_{k}\right\|$ and $\left\|\Sigma_{k=1}^{\infty} \xi_{k} u_{k}\right\|$ have finite expectations.

PROOF. Since the $\eta_{k}$ are uniformly nondegenerate, (5.19) implies that sup $\left\|u_{k}\right\| \leqslant c<\infty$ for some constant $c$. Since the $\eta_{k}$ are uniformly bounded we see from Theorem 3.3 that $E(M)<\infty$ and the condition on uniform nondegeneracy is equivalent to

$$
E\left(\left|\eta_{k}\right|\right)=\beta_{k}>\beta>0 \text { for some } \beta
$$


Let $\left\{\epsilon_{k}\right\}$ be a Rademacher sequence as in Remark 5.0. Notice that $\left\{\eta_{k} u_{k}\right\}$ and $\left\{\epsilon_{k}\left|\eta_{k}\right| u_{k}\right\}$ are stochastically similar. Hence (recall Remark 5.0)

$$
\begin{aligned}
E\left(\sup _{n}\left\|\sum_{k=1}^{n} \eta_{k} u_{k}\right\|\right) & =E_{2}\left(E_{1}\left(\sup _{n}\left\|\sum_{k=1}^{n} \epsilon_{k}\left|\eta_{k}\right| u_{k}\right\|\right)\right) \\
& \geqslant E_{2}\left(\sup _{n}\left\|\sum_{k=1}^{n} \epsilon_{k} \beta_{k} u_{k}\right\|\right) .
\end{aligned}
$$

Since $E(M)<\infty,(5.22)$ implies

$$
E\left(\sup _{n}\left\|\sum_{k=1}^{n} \epsilon_{k} \beta_{k} u_{k}\right\|\right)<\infty
$$

By Lemma $5.7\left(\beta_{k} u_{k}\right.$ replacing $u_{k}, \epsilon_{k}$ replacing $\eta_{k}$, and $\lambda_{k}=\beta_{k}^{-1} \leqslant \beta^{-1}$ by (5.21)) we get

$$
E\left(\sup _{n}\left\|\sum_{k=1}^{n} \epsilon_{k} u_{k}\right\|\right)<\infty .
$$

To go from $\left\{\epsilon_{k}\right\}$ to $\left\{\xi_{k}\right\}$ in (5.24) we make essentially the same argument as above. By Lemma 5.7

$$
E_{2}\left(\sup _{n}\left\|\sum_{k=1}^{n} \epsilon_{k}\left|\xi_{k}\right| u_{k}\right\|\right) \leqslant c_{1} E_{2}\left(\sup _{n}\left\|\sum_{k=1}^{n} \epsilon_{k} u_{k}\right\|\right),
$$

where $\sup _{k}\left|\xi_{k}\right| \leqslant c_{1}$ a.s. $\left(P_{1}\right)$. Using (5.24) and taking expectation with respect to $P_{1}$ in (5.25) shows that $E\left(M^{\prime}\right)<\infty$, which of course implies (5.26). This finishes the proof of the first part of the lemma.

If $\Sigma_{k=1}^{\infty} \eta_{k} u_{k}$ converges a.s., then we can complete the proof of the lemma if we show that $\Sigma_{k=1}^{\infty} \xi_{k} u_{k}$ converges a.s. The reason being that we can then use Lemma 2.1 to show that $M$ and $M^{\prime}$ are finite a.s. We have just proved that this implies $M$ and $M^{\prime}$ have finite expectations, which in turn implies, by the dominated convergence theorem, that $\left\|\sum_{k=1}^{\infty} \eta_{k} u_{k}\right\|$ and $\left\|\Sigma_{k=1}^{\infty} \xi_{k} u_{k}\right\|$ have finite expectations.

Assume that $\sum_{k=1}^{\infty} \eta_{k} u_{k}$ converges a.s., as above, the fact that the $\eta_{k}$ are uniformly nondegenerate implies $\left\|u_{k}\right\| \leqslant c<\infty$ uniformly in $k$. It follows from Theorem 3.3 that

$$
\lim _{n \rightarrow \infty} E\left(\left\|\sum_{k=n}^{\infty} \eta_{k} u_{k}\right\|\right)=0
$$

By Lemma 2.1 this implies (via integration by parts) that

$$
\lim _{n \rightarrow \infty} E\left(\sup _{m>n}\left\|\sum_{k=n}^{m} \eta_{k} u_{k}\right\|\right)=0 .
$$

Introducing $\left\{\epsilon_{k}\right\}$ as in Remark 5.0 and writing $\beta_{k}=E\left|\eta_{k}\right|$, the argument that leads to (5.23) shows that (5.27) implies 


$$
\lim _{n \rightarrow \infty} E\left(\sup _{m \geqslant n}\left\|\sum_{k=n}^{m} \epsilon_{k} \beta_{k} u_{k}\right\|\right)=0 .
$$

Since (5.21) still applies, we use Lemma 5.7 with $\lambda_{k}=\left|\xi_{k}(\omega)\right| \leqslant c_{1} \beta^{-1}$, where $\left|\xi_{k}(\omega)\right| \leqslant c_{1}$ a.s. for all $k$, and get

$$
E\left(\sup _{m \geqslant n}\left\|\sum_{k=n}^{m} \epsilon_{k} \beta_{k} u_{k}\right\|\right) \geqslant \beta c_{1}^{-1} E\left(\sup _{m \geqslant n}\left\|\sum_{k=n}^{m} \epsilon_{k}\left|\xi_{k}\right| u_{k}\right\|\right),
$$

therefore by (5.28) we have

$$
\lim _{n \rightarrow \infty} E\left(\sup _{m \geqslant n}\left\|\sum_{k=n}^{m} \xi_{k} u_{k}\right\|\right)=0 .
$$

Hence there exists a sequence of integers $\eta_{k}>\infty$ such that

$$
P\left(\sup _{m \geqslant n_{k}}\left\|\sum_{k=n_{k}}^{m} \xi_{k} u_{k}\right\|>2^{-k}\right) \leqslant 2^{-k} .
$$

This implies that $\sum_{k=1}^{\infty} \xi_{k} u_{k}$ converges a.s. by the Borel-Cantelli lemma. The proof of the lemma is complete.

LEMMA 5.10. Let $\left\{u_{k}\right\}$ be a sequence of elements in $B$ and $\left\{\eta_{k}\right\}$ be a sequence of independent, symmetric, real-valued random variables on $(\Omega, F, P)$. Let $\left\{\xi_{k}\right\}$ be a sequence of independent, symmetric, real-valued random variables on $(\Omega, F, P)$ satisfying

$$
P\left[\left|\eta_{k}\right| \geqslant x\right] \geqslant P\left[\left|\xi_{k}\right| \geqslant x\right]
$$

for all $x \geqslant 0$. Then

$$
\sup _{n}\left\|\sum_{k=1}^{n} \eta_{k} u_{k}\right\|<\infty \text { a.s. } \Rightarrow \sup _{n}\left\|\sum_{k=1}^{n} \xi_{k} u_{k}\right\|<\infty \text { a.s. }
$$

and

$$
\sum_{k=1}^{\infty} \eta_{k} u_{k} \text { converges a.s. } \Rightarrow \sum_{k=1}^{\infty} \xi_{k} u_{k} \text { converges a.s. }
$$

Proof. The main idea in the proof is to define $\left\{\eta_{k}\right\}$ and $\left\{\xi_{k}\right\}$ on the same probability space in such a way that $\left|\xi_{k}\right| \leqslant\left|\eta_{k}\right|$ a.s. and the finite-dimensional distributions of each sequence are preserved. Let $F_{k}(x)=P\left[\left|\eta_{k}\right| \leqslant x\right], G_{k}(x)=$ $P\left[\left|\xi_{k}\right| \leqslant x\right]$, and for $y \in[0,1]$, let $f_{k}(y)=\inf \left\{x: F_{k}(x) \geqslant y\right\}, g_{k}(y)=\inf \{x:$ $\left.G_{k}(x) \geqslant y\right\}$. In Remark 5.0 let $\left(\Omega_{1}, F_{1}, P_{1}\right)$ be such that on it are defined a Rademacher sequence $\left\{\epsilon_{k}^{\prime}\right\}$ and a sequence of independent random variables $\left\{\psi_{k}\right\}$, which is independent of $\left\{\epsilon_{k}^{\prime}\right\}$, such that, for $0 \leqslant u \leqslant 1, k \geqslant 1, P_{1}\left(\psi_{k} \leqslant u\right)=u$. We then define, for $k \geqslant 1, \widetilde{\eta}_{k}=f_{k}\left(\psi_{k}\right), \widetilde{\xi}_{k}=g_{k}\left(\psi_{k}\right)$. Clearly $\left\{\epsilon_{k}^{\prime} \widetilde{\eta}_{k}\right\}$ is sto- 
chastically equivalent to $\left\{\eta_{k}\right\}$ and $\left\{\epsilon_{k}^{\prime} \widetilde{\xi}_{k}\right\}$ stochastically equivalent to $\left\{\xi_{k}\right\}$. Furthermore, given $k \geqslant 1$ we have by (5.29), $f_{k}(x) \geqslant g_{k}(x)$ and $\left|\epsilon_{k}^{\prime} \tilde{\xi}_{k}\right|=\left|\tilde{\xi}_{k}\right| \leqslant\left|\tilde{\eta}_{k}\right|=$ $\left|\epsilon_{k}^{\prime} \tilde{\eta}_{k}\right|$ a.s. Hence, without loss of generality, we may assume that $\left\{\eta_{k}\right\}$ and $\left\{\xi_{k}\right\}$ are defined on $\left(\Omega_{1}, F_{1}, P_{1}\right)$ and for $k \geqslant 1$

$$
\left|\xi_{k}\right| \leqslant\left|\eta_{k}\right| \text { a.s. }
$$

Let $\left\{\epsilon_{k}\right\}$ be a Rademacher sequence on $\left(\Omega_{2}, F_{2}, P_{2}\right)$ and let $(\Omega, F, P)=\left(\Omega_{1} \times \Omega_{2}\right.$, $\left.F_{1} \times F_{2}, P_{1} \times P_{2}\right)$ as in Remark 5.0. We then have

$$
\sup _{n}\left\|\sum_{k=1}^{n} \eta_{k} u_{k}\right\|<\infty \text { a.s. } \Rightarrow \sup _{n}\left\|\sum_{k=1}^{n} \epsilon_{k} \eta_{k} u_{k}\right\|<\infty \text { a.s. }
$$

Hence for almost all $\omega_{1} \in \Omega_{1}$ (with respect to $P_{1}$ ),

$$
\sup _{n}\left\|\sum_{k=1}^{n} \epsilon_{k} \eta_{k}\left(\omega_{1}\right) u_{k}\right\|<\infty \text { a.s. }
$$

We now apply Lemma 5.9 with $\eta_{k}=\epsilon_{k}, u_{k}$ replaced by $\eta_{k}\left(\omega_{1}\right) u_{k}$ and $\xi_{k}$ replaced by $\epsilon_{k} \xi_{k}\left(\omega_{1}\right) \eta_{k}\left(\omega_{1}\right)^{-1}\left(=0\right.$ if $\left.\eta_{k}\left(\omega_{1}\right)=\xi_{k}\left(\omega_{1}\right)=0\right)$. Since $\xi_{k}$ is symmetric and uniformly bounded, by (5.32) we conclude that

$$
\sup _{n}\left\|\sum_{k=1}^{n} \epsilon_{k} \xi_{k}\left(\omega_{1}\right) u_{k}\right\|<\infty \text { a.s. }\left(P_{2}\right)
$$

for almost all $\omega_{1} \in \Omega_{1}\left(P_{1}\right)$. This shows (5.30). The proof for (5.31) is similar.

Proof of Theorem 5.1. To begin let us assume that $x_{0}=0$ in (5.5).

Let $\left(\Omega_{2}, F_{2}, P_{2}\right)$ be a probability space on which are defined a Rademacher sequence $\left\{\epsilon_{n}\right\}$ and a sequence $\left\{\alpha_{k}\right\}$, independent of $\left\{\epsilon_{n}\right\}$, of independent, identically distributed random variables such that $P_{2}\left(\alpha_{1}=1\right)=\alpha, P_{2}\left(\alpha_{1}=0\right)=1-\alpha$. Note that (Remark 5.0) $\left\{\eta_{k}\right\}$ and $\left\{\xi_{k}\right\}$ are on $\left(\Omega_{1}, F_{1}, P_{1}\right)$. Form the product space $\left(\Omega_{1} \times \Omega_{2}, F_{1} \times F_{2}, P_{1} \times P_{2}\right)$, so that $\left\{\eta_{k}\right\}$ and $\left\{\xi_{k}\right\}$ are independent of $\left\{\epsilon_{k}\right\}$ and $\left\{\alpha_{k}\right\}$. Let $\xi_{k}^{\prime}=\alpha_{k} \xi_{k}, k \geqslant 1$. We have, for $x>0, P\left[\left|\xi_{k}^{\prime}\right| \geqslant x\right]=$ $\alpha P\left[\left|\xi_{k}\right| \geqslant x\right]$. Therefore by (5.5) we get (with $x_{0}=0$ ) $P\left[\left|\eta_{k}\right| \geqslant x\right] \geqslant P\left[\left|\xi_{k}^{\prime}\right| \geqslant x\right]$ for all $x \geqslant 0$. Assume $\sup _{n}\left\|\Sigma_{k=1}^{n} \eta_{k} u_{k}\right\|<\infty$ a.s. By Lemma 5.10 this implies

$$
\sup _{n}\left\|\sum_{k=1}^{n} \alpha_{k} \xi_{k} u_{k}\right\|<\infty \text { a.s. }
$$

Since $\left\{\alpha_{k} \xi_{k}\right\}$ and $\left\{\epsilon_{k} \alpha_{k}\left|\xi_{k}\right|\right\}$ are stochastically equivalent, we may replace $\alpha_{k} \xi_{k}$ by $\epsilon_{k} \alpha_{k}\left|\xi_{k}\right|$ in (5.33) and by Fubini's theorem for almost all $\omega_{1} \in \Omega_{1}$ (with respect to $P_{1}$ ) 


$$
\sup _{n}\left\|\sum_{k=1}^{n} \epsilon_{k} \alpha_{k}\left|\xi_{k}\left(\omega_{1}\right)\right| u_{k}\right\|<\infty \text { a.s. }\left(P_{2}\right) \text {. }
$$

Since $\left\{\alpha_{k} \epsilon_{k}\right\}$ is uniformly nondegenerate, we apply Lemma 5.9 with $\eta_{k}=\alpha_{k} \epsilon_{k}$, $\left|\xi_{k}\left(\omega_{1}\right)\right| u_{k}$ for $u_{k}$ and $\epsilon_{k}$ for $\xi_{k}$ to conclude that

$$
\sup _{n}\left\|\sum_{k=1}^{n} \epsilon_{k}\left|\xi_{k}\left(\omega_{1}\right)\right| u_{k}\right\|<\infty \text { a.s. }\left(P_{2}\right)
$$

for almost all $\omega_{1} \in \Omega_{1}$. Hence

$$
\sup _{n}\left\|\sum_{k=1}^{n} \xi_{k} u_{k}\right\|<\infty \text { a.s. }
$$

This proves (5.6) when $x_{0}=0$ in (5.5).

If $x_{0}>0$ in (5.5), let

$$
\zeta_{k}=\left\{\begin{array}{ll}
\eta_{k} & \text { if }\left|\eta_{k}\right|>x_{0}, \\
0 & \text { otherwise; }
\end{array} \quad \zeta_{k}^{\prime}=\left\{\begin{array}{cl}
x_{0} & \text { if } 0<\eta_{k} \leqslant x_{0}, \\
-x_{0} & \text { if }-x_{0} \leqslant \eta_{k}<0, \\
0 & \text { otherwise. }
\end{array}\right.\right.
$$

By (5.5) we have $P\left[\left|\zeta_{k}+\zeta_{k}^{\prime}\right| \geqslant x\right] \geqslant \alpha P\left[\left|\xi_{k}\right| \geqslant x\right]$ for all $x \geqslant 0$. So if we can show that $\sup _{n}\left\|\Sigma_{k=1}^{n} \eta_{k} u_{k}\right\|<\infty$ a.s. implies

$$
\sup _{n}\left\|\sum_{k=1}^{n}\left(\zeta_{k}+\zeta_{k}^{\prime}\right) u_{k}\right\|<\infty
$$

then we will have by what we showed above that

$$
\sup _{n}\left\|\sum_{k=1}^{n} \xi_{k} u_{k}\right\|<\infty \text { a.s. }
$$

Assume $\sup _{n}\left\|\Sigma_{k=1}^{n} \eta_{k} u_{k}\right\|<\infty$ a.s. Since $P\left[\left|\eta_{k}\right| \geqslant x\right] \geqslant \alpha P\left[\left|\zeta_{k}\right| \geqslant x\right]$ for all $x \geqslant 0$, we have

$$
\sup _{n}\left\|\sum_{k=1}^{n} \zeta_{k} u_{k}\right\|<\infty \text { a.s. }
$$

Hence it is enough to show that

$$
\sup _{n}\left\|\sum_{k=1}^{n} \zeta_{k}^{\prime} u_{k}\right\|<\infty \text { a.s. }
$$

For this we use the uniform nondegeneracy of the $\eta_{k}$ 's. Let $a, b$ be numbers for which (5.3) holds. Let 


$$
\zeta_{k}^{\prime \prime}=\left\{\begin{aligned}
a & \text { if } \eta_{k} \geqslant a, \\
-a & \text { if } \eta_{k} \leqslant-a \\
0 & \text { otherwise. }
\end{aligned}\right.
$$

Now the $\zeta_{k}^{\prime \prime}$ are independent, symmetric random variables and (by (5.3)) $P\left[\left|\eta_{k}\right| \geqslant x\right]$ $\geqslant b P\left[\left|\zeta_{k}^{\prime \prime}\right| \geqslant x\right]$ for all $x \geqslant 0$; hence again by the special case considered above we have

$$
\sup _{n}\left\|\sum_{k=1}^{n} \zeta_{k}^{\prime \prime} u_{k}\right\|<\infty \text { a.s. }
$$

Since the $\zeta_{k}^{\prime \prime}$ are also uniformly nondegenerate and uniformly bounded, we now conclude by Lemma 5.9 that (5.41) implies (5.40). This proves (5.6). The assertion (5.7) follows by similar arguments which are omitted.

We need the following extension of Lemma $5.7(5.16)$ in the proof of Theorem 5.3.

LEMmA 5.11. Given the hypotheses of Lemma 5.7 assume that $\Sigma_{k=1}^{\infty} \eta_{k} u_{k}$ converges a.s. Then $\sum_{k=1}^{\infty} \lambda_{k} \eta_{k} u_{k}$ converges a.s. and

$$
E\left[\varphi\left(\left\|\sum_{k=1}^{\infty} \lambda_{k} \eta_{k} u_{k}\right\|\right)\right] \leqslant E\left[\varphi\left(c\left\|\sum_{k=1}^{\infty} \eta_{k} u_{k}\right\|\right)\right] \text {. }
$$

Proof. Since $\Sigma_{k=1}^{\infty} c \eta_{k} u_{k}$ converges a.s., by Theorem $5.1 \sum_{k=1}^{\infty} \lambda_{k} \eta_{k} u_{k}$ converges a.s. because $P\left[\left|c \eta_{k}\right| \geqslant x\right] \geqslant P\left[\left|\lambda_{k} \eta_{k}\right| \geqslant x\right]$ for all $x \geqslant 0$. Once we know that both series converge a.s. we can use the proof of Lemma 5.7 with $\left\|\Sigma_{k=1}^{\infty}\right\|$ replacing $\sup _{n}\left\|\Sigma_{k=1}^{n}\right\|$.

Proof of Theorem 5.3. Case (i). $x_{0}=0, \alpha=1$. As in the proof of Lemma 5.10 we can assume that both $\left\{\eta_{k}\right\}$ and $\left\{\xi_{k}\right\}$ are defined on the same probability space $\left(\Omega_{1}, F_{1}, P_{1}\right)$ and that, for all $k \geqslant 1,\left|\eta_{k}\right| \geqslant\left|\xi_{k}\right|$ a.s. We define a Rademacher sequence $\left\{\epsilon_{n}\right\}$ on a probability space $\left(\Omega_{2}, F_{2}, P_{2}\right)$ and form the product space $\left(\Omega_{1} \times \Omega_{2}, F_{1} \times F_{2}, P_{1} \times P_{2}\right)$ as before. We have

$$
E\left[\varphi\left(\sup _{n}\left\|\sum_{k=1}^{n} \eta_{k} u_{k}\right\|\right)\right]=E_{1}\left[E_{2}\left\{\varphi\left(\sup _{n}\left\|\sum_{k=1}^{n} \epsilon_{k}\left|\eta_{k}\left(\omega_{1}\right)\right| u_{k}\right\|\right)\right\}\right] \text {, }
$$

where $\omega_{1} \in \Omega_{1}$ is held fixed when $E_{2}$ operates. We now apply Lemma 5.7 (5.16) with $\lambda_{k}=\left|\xi_{k}\left(\omega_{1}\right) \| \eta_{k}\left(\omega_{1}\right)\right|^{-1}\left(=0\right.$ if $\left.\xi_{k}\left(\omega_{1}\right)=\eta_{k}\left(\omega_{1}\right)=0\right)$. Since $\left|\lambda_{k}\right| \leqslant 1$ we see that the right-hand side in (5.42) dominates

$$
E_{1}\left[E_{2}\left\{\varphi\left(\sup _{n}\left\|\sum_{k=1}^{n} \epsilon_{k}\left|\xi_{k}\left(\omega_{1}\right)\right| u_{k}\right\|\right)\right\}\right]=E\left[\varphi\left(\sup _{n}\left\|\sum_{k=1}^{n} \xi_{k} u_{k}\right\|\right)\right] .
$$

This proves Case (i).

Case (ii). $x_{0}=0,0<\alpha \leqslant 1$. Let independent random variables $\alpha_{k}, k \geqslant 1$, 
be defined on $\left(\Omega_{2}, F_{2}, P_{2}\right)$ such that $P_{2}\left(\alpha_{k}=1\right)=\alpha, P_{2}\left(\alpha_{k}=0\right)=1-\alpha$, $k \geqslant 1$, and let $\xi_{k}^{\prime}=\alpha_{k} \xi_{k}$. Since, for all $x \geqslant 0, P\left[\left|\eta_{k}\right| \geqslant x\right] \geqslant P\left[\left|\xi_{k}^{\prime}\right| \geqslant x\right]$ we apply Case (i) to get $\left(E_{2}\left(\alpha_{k}\right)=\alpha\right)$

$$
\begin{gathered}
E\left[\varphi\left(\sup _{n} \alpha^{-1}\left\|\sum_{k=1}^{n} \eta_{k} u_{k}\right\|\right)\right] \geqslant E\left[\varphi\left(\sup _{n} \alpha^{-1}\left\|\sum_{k=1}^{n} \xi_{k}^{\prime} u_{k}\right\|\right)\right] \\
=E_{1}\left[E_{2}\left(\varphi\left(\sup _{n} \alpha^{-1}\left\|\sum_{k=1}^{n} \alpha_{k} \xi_{k} u_{k}\right\|\right)\right)\right] \\
\geqslant E_{1}\left[\varphi\left(\sup _{n} \alpha^{-1}\left\|\sum_{k=1}^{n} E_{2}\left(\alpha_{k}\right) \xi_{k} u_{k}\right\|\right)\right] \\
=E_{1}\left[\varphi\left(\sup _{n}\left\|\sum_{k=1}^{n} \xi_{k} u_{k}\right\|\right)\right],
\end{gathered}
$$

where Jensen's inequality applies since $\varphi$ is convex. This completes the proof of (5.8). The assertion after (5.8) follows similarly by using Lemma 5.11 in place of Lemma 5.7.

We now consider the case $x_{0}>0$. Define $\zeta_{k}$ and $\zeta_{k}^{\prime}$ as in the proof of Theorem 5.1. Then for all $x \geqslant 0$ we have

$$
P\left[\left|\zeta_{k}+\zeta_{k}^{\prime}\right| \geqslant x\right] \geqslant \alpha P\left[\left|\xi_{k}\right| \geqslant x\right] .
$$

Applying the first part of this theorem $\left(x_{0}=0\right)$, we get

$$
E\left[\varphi\left(\sup _{n}\left\|\sum_{k=1}^{n} \xi_{k} u_{k}\right\|\right)\right] \leqslant E\left[\varphi\left(\alpha^{-1} \sup _{n}\left\|\sum_{k=1}^{n}\left(\zeta_{k}+\xi_{k}^{\prime}\right) u_{k}\right\|\right)\right]
$$

and using the facts that $\varphi$ is nondecreasing and convex, we dominate the right side in (5.44) by

$$
\frac{1}{2} E\left[\varphi\left(\frac{2}{\alpha} \sup _{n}\left\|\sum_{k=1}^{n} \zeta_{k} u_{k}\right\|\right)\right]+\frac{1}{2} E\left[\varphi\left(\frac{2}{\alpha} \sup _{n}\left\|\sum_{k=1}^{n} \zeta_{k}^{\prime} u_{k}\right\|\right)\right] .
$$

Since $\left|\zeta_{k}\right| \leqslant\left|\eta_{k}\right|$, and $\left|\zeta_{k}^{\prime}\right| \leqslant x_{0}$, applying (5.8) to each part in the sum (5.45) we dominate $(5.45)$ by

$$
\frac{1}{2} E\left[\varphi\left(\frac{2}{\alpha} \sup _{n}\left\|\sum_{k=1}^{n} \eta_{k} u_{k}\right\|\right)\right]+\frac{1}{2} E\left[\varphi\left(\frac{2 x_{0}}{\alpha} \sup _{n}\left\|\sum_{k=1}^{n} \epsilon_{k} u_{k}\right\|\right)\right],
$$

where $\left\{\epsilon_{k}\right\}$ is a Rademacher sequence. (We know that $\sup _{n}\left\|\Sigma_{k=1}^{n} \epsilon_{k} u_{k}\right\|<\infty$ a.s. by Corollary 5.2.) Let $\delta=2 x_{0} \alpha^{-1}$. To get (5.9) it remains to check that

$$
E\left[\varphi\left(\delta \sup _{n}\left\|\sum_{k=1}^{n} \epsilon_{k} u_{k}\right\|\right)\right] \leqslant E\left[\varphi\left(\frac{\delta}{a b} \sup _{n}\left\|\sum_{k=1}^{n} \eta_{k} u_{k}\right\|\right)\right] .
$$


But this follows by observing that (5.3) implies $P\left[\left|\eta_{k} a^{-1}\right| \geqslant x\right] \geqslant b P\left[\left|\epsilon_{k}\right| \geqslant x\right]$ for all $x \geqslant 0, k \geqslant 1$.

For the analogue of (5.9) when $\Sigma_{k=1}^{\infty} \eta_{k} u_{k}$ converges a.s. we use the same proof, this time working with the assertion following (5.8). This completes the proof of Theorem 5.3.

The proofs for general $\eta_{k}$ follow easily from the following lemma.

LEMMA 5.12. Let $\eta$ be a real-valued random variable satisfying (5.10) with $\gamma$ and $\delta$, and let $\eta^{\prime}$ be an independent copy of $\eta$, then $P\left[\left|\eta-\eta^{\prime}\right| \geqslant x / 2\right] \geqslant$ $\delta P[|\eta| \geqslant x]$ for $x \geqslant 2 \gamma$.

Proof. By (5.10) and independence of $\eta, \eta^{\prime}$

$$
\delta P[|\eta| \geqslant x] \leqslant P\left[|\eta| \geqslant x,\left|\eta^{\prime}\right|<\gamma\right] \leqslant P\left[\left|\eta-\eta^{\prime}\right| \geqslant x-\gamma\right] \leqslant P\left[\left|\eta-\eta^{\prime}\right| \geqslant x / 2\right]
$$

for $x \geqslant 2 \gamma$.

The proofs of Theorems 5.4, 5.5 and 5.6 follow by symmetrizing $\left\{\eta_{k}\right\}$ and applying the corresponding result in the symmetric case with the help of Lemma 5.12. We will give the details only for the proof of Theorem 5.4.

Proof of TheOREM 5.4. Suppose $\sup _{n}\left\|\sum_{k=1}^{n} \eta_{k} u_{k}\right\|<\infty$ a.s. Let $\left\{\eta_{k}^{\prime}\right\}$ be an independent copy of $\left\{\eta_{k}\right\}$. Clearly

$$
\sup _{n}\left\|\sum_{k=1}^{n} 2\left(\eta_{k}-\eta_{k}^{\prime}\right) u_{k}\right\|<\infty \text { a.s. }
$$

The sequence $\left\{2\left(\eta_{k}-\eta_{k}^{\prime}\right)\right\}$ is symmetric and satisfies (5.3) for some $a>0$ and $b>0$. Also by Lemma 5.12 and (5.11), $P\left[\left|2\left(\eta_{k}-\eta_{k}^{\prime}\right)\right| \geqslant x\right] \geqslant \alpha \delta P\left[\left|\xi_{k}\right| \geqslant x\right]$ for $x \geqslant x_{0}$. We can apply Theorem 5.1 to $2\left(\eta_{k}-\eta_{k}^{\prime}\right)$ and $\left\{\xi_{k}\right\}$ to obtain $\sup _{n}\left\|\Sigma_{k=1}^{n} \xi_{k} u_{k}\right\|<\infty$ a.s. This proves (5.12). A similar proof applies to (5.13).

6. Applications. We will now apply the results of the previous sections to a variety of problems in Gaussian processes and series of random functions.

6(a). Gaussian processes. We will derive a theorem of Landau and Shepp [6].

Let $\left\{\varphi_{n}\right\}$ be a sequence of elements of $B$ and let $\left\{Y_{n}\right\}$ be a sequence of independent, identically distributed, random variables on $(\Omega, F, P)$. For $\omega \in \Omega$ we write as before

$$
S_{n}(\omega)=\sum_{j=1}^{n} \varphi_{j} Y_{j}(\omega)
$$

We then have

THEOREM 6.1. Suppose $M=\sup _{n}\left\|S_{n}\right\|<\infty$ a.s. If $Y_{1}$ is Gaussian with mean 0 , variance 1 , then there exists $\epsilon>0$ such that $E\left(\exp \left(\epsilon M^{2}\right)\right)<\infty$.

Proof. By applying Theorem 3.8 we will first show that $E(\exp (\epsilon M))<\infty$ for some $\epsilon>0$. Therefore by (3.25), taking $\delta=1$, we need to show that for 
some $\alpha>0, a>0$

$$
\sum_{n=1}^{\infty} \frac{1}{\left\|\varphi_{n}\right\|} \int_{a}^{\infty} \exp \left(\alpha u \log ^{2} u-\frac{u^{2}}{2\left\|\varphi_{n}\right\|^{2}}\right) d u<\infty .
$$

(Note that $\left\|\varphi_{n}\right\| \neq 0$ may be assumed for all $n$.) Since $M<\infty$ a.s. it follows from Remark 3.2 that

$$
\sum_{n=1}^{\infty} P\left(\left\|\varphi_{n}\right\|\left|Y_{n}\right|>\lambda\right)<\infty
$$

for some $\lambda<0$. Using the well-known Gaussian tail estimate (6.3) implies

$$
\sum_{n=1}^{\infty} \frac{1}{\left\|\varphi_{n}\right\|} \exp \left(-\lambda^{2} / 2\left\|\varphi_{n}\right\|^{2}\right)<\infty .
$$

For $a \geqslant 1$ the sum in (6.2) is dominated by

$$
\sum_{n=1}^{\infty} \frac{1}{\left\|\varphi_{n}\right\|} \int_{a}^{\infty} \exp \left\{-\frac{u^{2}}{2\left\|\varphi_{n}\right\|^{2}}\left(1-\alpha\left\|\varphi_{n}\right\|^{2}\right)\right\} d u
$$

and since $\left\|\varphi_{n}\right\|$ must be bounded, the sum in (6.5) converges for suitable $\alpha>0$ by (6.4). Hence we have for some $\epsilon>0$

$$
E(\exp (\epsilon M))<\infty
$$

The proof of the theorem is now completed by the following argument which was shown to us by S. R. S. Varadhan.

Let $\left\{Y_{n, j}, n \geqslant 1\right\}, j \geqslant 1$, be independent copies of the sequence $\left\{Y_{n}, n \geqslant 1\right\}$, and let $S_{n, j}=\sum_{k=1}^{n} \varphi_{k} Y_{k, j}$. Then for $a>0, m$ a positive integer

$$
P\left(\left\|S_{n}\right\|>a \sqrt{ } m\right)=P\left(\left\|\frac{S_{n, 1}+\cdots+S_{n, m}}{\sqrt{ } m}\right\|>a \sqrt{ } m\right),
$$

by noting that the $Y_{n, j}$ 's are independent $N(0,1)$ and hence $S_{n}$ has the same distribution as $\left(S_{n, 1}+\ldots+S_{n, m}\right) m^{-1 / 2}$. Using Chebychev's inequality we get

$$
P\left(\left\|S_{n, 1}+\cdots+S_{n, m}\right\|>a m\right) \leqslant\left[E\left(\exp \left(\epsilon\left\|S_{n}\right\|\right)\right)\right]^{m} \exp (-\epsilon a m)
$$

Since $\left\|S_{n}\right\| \leqslant M$, combining (6.6), (6.7) and (6.8) we get

$$
P\left(\left\|S_{n}\right\|>a \sqrt{ } m\right) \leqslant[E(\exp (\epsilon M))]^{m} \exp (-\epsilon a m)
$$

where $\epsilon$ does not depend on $n$. Hence by taking $a$ sufficiently large, we get $0<$ $\rho<1$, independent of $k$, such that, for $m \geqslant 1$,

$$
P\left(\left\|S_{n}\right\|>a \sqrt{ } m\right) \leqslant \rho^{m} \text {. }
$$

This implies that $\exists \epsilon_{1}>0, A<\infty$, independent of $n$, such that 


$$
E\left(\exp \left(\epsilon_{1}\left\|S_{n}\right\|^{2}\right)\right) \leqslant A
$$

Since $S_{n}$ are symmetric, by Lemma $2.1,(6.11)$ gives

$$
E\left(\exp \left(\epsilon_{1}\left\|M_{n}\right\|^{2}\right)\right) \leqslant 2 A .
$$

The result now follows by the monotone convergence theorem.

The following corollary of the above theorem was proved by Landau and Shepp [6]. For a more general setting and a different proof see Fernique [2] (see also [9]).

COROLLARY 6.2. Let $\left\{X_{n}, n \geqslant 1\right\}$ be an arbitrary sequence of real-valued Gaussian random variables on $(\Omega, F, P)$. Let $Z=\sup _{n}\left|X_{n}\right|$. Then

$$
Z<\infty \text { a.s. } \Rightarrow E\left(\exp \left(\epsilon Z^{2}\right)\right)<\infty
$$

for some $\epsilon>0$.

Proof. Let $T=\{1,2, \ldots\}$ with the discrete metric, i.c. $d(i, j)=1(0)$ for $i \neq j(i=j), d$ denoting the metric. Take $B=C(T)=$ all real bounded sequences with the norm on $B$ the sup norm. We can use the Gram-Schmidt process, and for each $n \in T$, write

$$
X_{n}=\sum_{j=1}^{\infty} \varphi_{j}(n) Y_{j},
$$

where the $Y_{j}$ are mutually orthogonal $N(0,1)$ random variables, and $\varphi_{j}(n)$ fixed real numbers determined by the joint distributions of the $X_{n}$, and

$$
E\left(X_{n}^{2}\right)=\sum_{j=1}^{\infty} \varphi_{j}(n)^{2}
$$

Since the $Y_{j}$ 's are uncorrelated and Gaussian, they are mutually independent.

Hence the series in (6.13) converges a.s. for each $n$. By Lemma 2.1, for all $a>0$ and $N$ positive integer,

$$
P\left(\sup _{k>1} \sup _{1<n<N}\left|\sum_{j=1}^{k} \varphi_{j}(n) Y_{j}\right|>a\right) \leqslant 2 P\left(\sup _{1 \leqslant n \leqslant N} \cdot\left|\sum_{j=1}^{\infty} \varphi_{j}(n) Y_{j}\right|>a\right) .
$$

Letting $N \rightarrow \infty$, we have for all $a>0$

$$
P\left(\sup _{N>1} \sup _{k>1} \sup _{1<n<N}\left|\sum_{j=1}^{\infty} \varphi_{j}(n) Y_{j}\right|>a\right) \leqslant 2 P\left(\sup _{N \geqslant 1}\left|X_{N}\right|>a\right),
$$

and since $\sup _{N>1}\left|X_{N}\right|=Z<\infty$ a.s. by assumption, we have

$$
\sup _{N>1} \sup _{k \geqslant 1} \sup _{1<n<N}\left|\sum_{j=1}^{k} \varphi_{j}(n) Y_{j}\right|<\infty \text { a.s. }
$$

The expression in (6.15) equals $\sup _{k>1} \sup _{n \geqslant 1}\left|\Sigma_{j=1}^{k} \varphi_{j}(n) Y_{j}\right|=M$, in the notation of Theorem 6.1. Hence $Z<\infty$ a.s. implies $M<\infty$ a.s. and Theorem 6.1 applies 
to give an $\epsilon>0$ such that $E\left(\exp \left(\epsilon M^{2}\right)\right)<\infty$; since $Z \leqslant M$, the corollary follows.

6(b). Sums of independent Banach space valued random variables. The following theorem is an immediate corollary of Corollary 3.5.

THEOREM 6.3. Let $\left\{X_{k}\right\}$ be independent random variables with values in a separable Banach space $B$. Let $\left\{a_{k}\right\} \in l^{p}, 0<p<\infty$. Assume that $\Sigma a_{k} X_{k}$ converges a.s. and that $E\left(\left\|X_{k}\right\|^{p}\right) \leqslant c<\infty$ for all $k$. Then $E\left(\left\|\Sigma a_{k} X_{k}\right\|^{p}\right)<\infty$.

6(c). Random Fourier series. For $0 \leqslant t \leqslant 2 \pi$ consider

$$
\xi(t)=\sum_{n=0}^{\infty} a_{n} \eta_{n} \cos \left(n t+\Phi_{n}\right)
$$

where $\left\{\eta_{n} e^{i \Phi_{n}}\right.$ \} is a sequence of independent complex valued random variables ( $\eta_{n}$ and $\Phi_{n}$ are real random variables); $E\left(\eta_{n}^{2}\right) \equiv 1$ and $\left\{a_{n}\right\} \in l^{2}$. Since $\left\{a_{n}\right\} \in l^{2}$, for a fixed $t$ the sum in (6.16) converges a.s. In the study of these series it is natural to require that $\left\{a_{n}\right\} \in l^{2}$.

THEOREM 6.4. Assume that the series in (6.16) converges uniformly a.s. and let $\|\xi\|=\sup _{t}|\xi(t)|$. Then

(i) $E\left(\left|\eta_{n}\right|^{p}\right) \leqslant c<\infty, 2 \leqslant p<\infty$, implies $E\left(\|\xi\|^{p}\right)<\infty$.

(ii) $P\left(\left|\eta_{n}\right|>u\right) \leqslant \exp \left(-\alpha u \log ^{1+\delta} u\right)$ for some $\alpha>0, \delta>0$, and all $u \geqslant 1$ implies $E(\exp (\epsilon\|\xi\|))<\infty$ for some $\epsilon>0$.

(iii) $\left|\eta_{n}\right| \leqslant A<\infty$ a.s. implies $E(\exp (\lambda\|\xi\|))<\infty$ for all $\lambda<\infty$.

Proof. (i) follows from Theorem 6.3, (ii) from Theorem 3.8 and (iii) from Theorem 3.11 .

REMARK. An extension of Theorem 6.4 (iii), under additional hypotheses, is given in $\S 6(d)$.

We now consider the question of whether the series in (6.16) converge uniformly a.s. To simplify the discussion we will assume that $\eta_{n} e^{i \Phi_{n}}, n=0,1, \ldots$, are also symmetric. Let

$$
\begin{aligned}
& \sigma^{2}(h)=\sum_{n=0}^{\infty} a_{n}^{2} \sin ^{2} \frac{n h}{2}, \\
& \bar{\sigma}^{2}(h)=\text { nondecreasing rearrangement of } \sigma^{2}(h), \quad h \in[0,2 \pi],
\end{aligned}
$$

(see [4] for exact definition) and

$$
I(\bar{\sigma})=\int_{0}^{1 / 2} \frac{\bar{\sigma}(h)}{h(\log 1 / h)^{1 / 2}} d h .
$$

If $\left\{\eta_{n}\right\}$ are independent Gaussian random variables with $E \eta_{n}=0, E \eta_{n}^{2}=1$ then $I(\bar{\sigma})<\infty$ if and only if the series in (6.16) converges uniformly a.s. (see [7, §3] for further discussion and references). In [7] it is also shown that $I(\bar{\sigma})<\infty$ implies that the series in (6.16) converges uniformly a.s. if the $\eta_{k}$ are sub-Gaussian; 
however, as far as we know, no inference has been made about the behavior of (6.16) for non-Gaussian $\eta_{k}$ when $I(\bar{\sigma})=\infty$. We can do this readily by applying Theorem 5.1.

THEOREM 6.5. Consider the series in (6.16) with the additional assumption that $\eta_{n} e^{i \Phi_{n}}, n=0,1, \ldots$, are symmetric. Suppose there exist an $\alpha>0$ and $x_{0}$ such that

$$
P\left[\left|\eta_{k}\right| \geqslant x\right] \geqslant \alpha \int_{x}^{\infty} e^{-u^{2} / 2} d u
$$

for all $x \geqslant x_{0}$. Then $I(\bar{\sigma})=\infty$ implies that the series in (6.16) is unbounded a.s.

Proof. For $\Phi_{n} \equiv 0$ the proof is an immediate consequence of Theorem 5.1 and the above mentioned result for Gaussian Fourier series. This result extends to the general symmetric $\eta_{n} e^{i \Phi_{n}}$ by Theorem 3.1 in [7]. The dichotomy between uniform convergence and unboundedness can be found in [5, p. 49].

6(d). Uniformly bounded random variables. By strengthening the hypothesis of Theorem 4.8 we prove the results referred to in Remark 4.9.

THEOREM 6.6. Let $\left\{\eta_{k}\right\}$ be independent standard Gaussian random variables and $\left\{u_{k}\right\}$ a sequence of elements in $B$. Let $\left\{\xi_{k}\right\}$ be independent symmetric realvalued random variables satisyfing $\sup _{k}\left|\xi_{k}\right| \leqslant c<\infty$ a.s. Then if $\Sigma_{k=1}^{\infty} \eta_{k} u_{k}$ converges a.s., so does $\Sigma_{k=1}^{\infty} \xi_{k} u_{k}$ and

$$
E\left(e^{\lambda\left\|\Sigma_{k=1}^{\infty} \xi k_{k}\right\|^{2}}\right)<\infty
$$

for all real $\lambda$.

Proof. The fact that $\Sigma_{k=1}^{\infty} \xi_{k} u_{k}$ converges a.s. follows immediately from Corollary 5.2. It follows from Fernique [2] that since $\Sigma_{k=1}^{\infty} \eta_{k} u_{k}$ converges a.s., there exist $\lambda_{n}>0$ such that

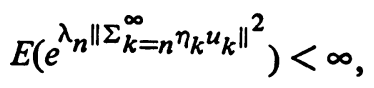

where $\lambda_{n} \rightarrow \infty$ as $n \rightarrow \infty$. If the $\xi_{k}$ are symmetric and satisfy (5.3) with $a, b$, let $v_{0}=\max (2,2 c / a b)$. Clearly $P\left[\left|\eta_{k}\right| \geqslant x\right]>P\left[\left|\xi_{k}\right| \geqslant x\right]$ for $x>c$. Given any real $\lambda$ we can pick $n$ sufficiently large by $(6.18)$ so that

$$
E\left[e^{2 v_{0}^{2} \lambda\left\|\Sigma_{k=n}^{\infty} \eta_{k} u_{k}\right\|^{2}}\right]<\infty .
$$

It follows from (5.9) that

$$
E\left[e^{2 \lambda\left\|\Sigma_{k=n}^{\infty} \xi_{k} u_{k}\right\|^{2}}\right]<\infty .
$$

Since $\sum_{k=1}^{\infty} \xi_{k} u_{k}$ converges a.s. implies that $\sup _{k}\left\|u_{k}\right\| \leqslant A$ (for some constant $A$ ), we have 


$$
E\left[e^{\lambda\left\|\Sigma_{k=1}^{n-1} \xi_{k} u\right\|^{2}}\right] \leqslant e^{\lambda n^{2} A^{2} c^{2}}<\infty
$$

and (6.19) and (6.20) give (6.17).

\section{REFERENCES}

1. W. Feller (1966), An introduction to probability theory and its applications. Vol. II, Wiley, New York. MR 35 \#1048.

2. X. Fernique (1970), Intégrabilité des vecteurs Gaussiens, C. R. Acad. Sci. Paris Sér. A-B 270, A1698-A1699. MR 42 \#1170.

3. J. Hoffmann-J $\phi r g e n s e n ~(1973)$, Sums of independent Banach space valued random variables, Aarhus Universitet Matematisk Institut, Denmark (preprint).

3a. - (1974), Sums of independent Banach space valued random variables, Studia Math. 52 (1974), 159-186.

4. N. C. Jain and M. B. Marcus (1974), Sufficient conditions for the continuity of stationary Gaussian processes and applications to random series of functions, Ann. Inst. Fourier (Grenoble) 24, 117-141.

5. J.-P. Kahane (1968), Some random series of functions, Heath, Lexington, Mass. MR 40 \#8095.

6. H. J. Landau and L. A. Shepp (1970), On the supremum of a Gaussian process, Sankhyā Ser. A 32, 369-378. MR 44 \#3381.

7. M. B. Marcus (1974), Uniform convergence of random Fourier series, Ark. Mat. (to appear).

8. G. Pisier (1973), Type des espaces normes, C. R. Acad. Sci. Paris Sér. A-B 276, A1673-A1676.

9. A. V. Skorohod (1970), A note on Gaussian measures in Banach space, Teor. Verojatnost. i Primenen. 15, 519-520. (Russian) MR 43 \#3417.

\section{MINNESOTA 55455}

DEPARTMENT OF MATHEMATICS, UNIVERSITY OF MINNESOTA, MINNEAPOLIS,

DEPARTMENT OF MATHEMATICS, NORTHWESTERN UNIVERSITY, EVANSTON, ILLINOIS 60201 